\title{
Gaia white dwarfs within 40 pc II: the volume-limited northern hemisphere sample
}

\author{
Jack McCleery, ${ }^{1 \star}$ Pier-Emmanuel Tremblay, ${ }^{1}$ Nicola Pietro Gentile Fusillo, ${ }^{2}$ \\ Mark A. Hollands, ${ }^{1}$ Boris T. Gänsicke, ${ }^{1}$ Paula Izquierdo, ${ }^{3,4}$ Silvia Toonen, ${ }^{5}$ \\ Tim Cunningham, ${ }^{1}$ and Alberto Rebassa-Mansergas ${ }^{6,7}$ \\ ${ }^{1}$ Department of Physics, University of Warwick, CV4 7AL, Coventry, UK \\ ${ }^{2}$ European Southern Observatory, Karl-Schwarzschild-Str. 2, D 85748 Garching, Germany \\ ${ }^{3}$ Instituto de Astrofísica de Canarias, 38205 La Laguna, Tenerife, Spain \\ ${ }^{4}$ Departamento de Astrofísca, Universidad de La Laguna, 38206 La Laguna,Tenerife, Spain \\ ${ }^{5}$ Institute for Gravitational Wave Astronomy, School of Physics and Astronomy, University of Birmingham, Birmingham, \\ B15 2TT, UK \\ ${ }^{6}$ Departament de Física, Universitat Politècnica de Catalunya, c/Esteve Terrades 5, 08860 Castelldefels, Spain \\ ${ }^{7}$ Institut d'Estudis Espacials de Catalunya, Ed. Nexus-201, c/Gran Capità 2-4, 08034 Barcelona, Spain
}

Accepted XXX. Received YYY; in original form ZZZ

\begin{abstract}
We present an overview of the sample of northern hemisphere white dwarfs within $40 \mathrm{pc}$ of the Sun detected from Gaia Data Release 2 (DR2). We find that 521 sources are spectroscopically confirmed degenerate stars, 111 of which were first identified as white dwarf candidates from Gaia DR2 and followed-up recently with the William Herschel Telescope and Gran Telescopio Canarias. Three additional white dwarf candidates remain spectroscopically unobserved and six unresolved binaries are known to include a white dwarf but were not in our initial selection in the Gaia DR2 Hertzsprung-Russell diagram (HRD). Atmospheric parameters are calculated from Gaia and Pan-STARRS photometry for all objects in the sample, confirming most of the trends previously observed in the much smaller $20 \mathrm{pc}$ sample. Local white dwarfs are overwhelmingly consistent with Galactic disc kinematics, with only four halo candidates. We find that DAZ white dwarfs are significantly less massive than the overall DA population $\left(\bar{M}_{\mathrm{DAZ}}=0.59 \mathrm{M}_{\odot}, \bar{M}_{\mathrm{DA}}=0.66 \mathrm{M}_{\odot}\right)$. It may suggest that planet formation is less efficient at higher mass stars, producing more massive white dwarfs. We detect a sequence of crystallised white dwarfs in the mass range from $0.6 \lesssim M / \mathrm{M}_{\odot} \lesssim 1.0$ and find that the vast majority of objects on the sequence have standard kinematic properties that correspond to the average of the sample, suggesting that their nature can be explained by crystallisation alone. We also detect 26 double degenerates and white dwarf components in 56 wide binary systems.
\end{abstract}

Key words: white dwarfs - stars: statistics - solar neighbourhood

\section{INTRODUCTION}

Stars are born in groups with initial spatial and kinematic homogeneity but large-scale galactic dynamical disturbances due to spiral arms and mergers greatly affect their present day orbits. The precise astrometric and photometric observations from the Gaia spacecraft (Gaia Collaboration et al. 2018a) have improved our understanding of the formation and chemical evolution of stars in the Milky Way (see, e.g.

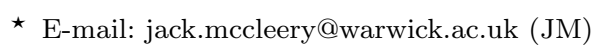

Haywood et al. 2018) and have recognised the role played by a merger with at least one satellite galaxy in the formation of the thick disc (Helmi et al. 2018). As these stars age, deplete their nuclear energy source and evolve as giants and white dwarfs, their luminosity can change by a factor of up to $10^{8}$. It has therefore been a challenge to assemble representative stellar samples of all ages and masses. Gaia DR2 has led to a major increase in the size of the local volume-limited sample, detecting the vast majority of main-sequence stars and white dwarfs within $\approx 100 \mathrm{pc}$ (Gaia Collaboration et al. 2018b; Gentile Fusillo et al. 2019). This constitutes a unique 
snapshot of stars that have formed at all look-back times and with initial masses from the hydrogen burning limit to $10 \mathrm{M}_{\odot}$. The detailed formation history of this present-day local stellar sample can help to constrain the overall formation history and radial migration of stars and their planets in the Milky Way (Minchev et al. 2013; Fantin et al. 2019).

While the advances made by Gaia DR2 have been transformative, identifying up to 400000 sources within 100 pc, a full understanding of the local stellar population is still a major challenge. The local Gaia HRD is contaminated by distant sources with erroneous parallaxes as well as faint sources with improper sky background subtraction, with the recommended quality cuts reducing the size of the local sample by a significant factor (Gaia Collaboration et al. 2018b). Furthermore most of the local Gaia sources do not have spectroscopic follow-up, with the spectral type completeness dropping to a small percentage beyond 20 pc (see, e.g., Henry et al. 2018). This largely prevents the determination of precise stellar parameters from the lack of atmospheric chemical abundances, magnetic field strengths and binary parameters. Furthermore, stellar modelling needs to be improved considering that currently employed stellar evolution tracks for white dwarfs and $\mathrm{M}$ dwarfs deviate from the empirical Gaia HRD (Hollands et al. 2018b; Parsons et al. 2018; Morrell \& Naylor 2019). These modelling issues directly impact the characterisation of the bulk properties of nearby exoplanets around M dwarfs and evolved planetary systems at white dwarfs.

Surveys of nearby cool white dwarfs have historically used reduced proper motion as a proxy for distance coupled with targeted spectroscopic and astrometric follow-ups (Liebert et al. 1988; Bergeron et al. 1997; Limoges et al. 2015; Subasavage et al. 2017). Over the time, white dwarfs likely to be within $20-25$ pc were catalogued in a series of papers which highlighted the diversity of the local stellar remnant population and their space density (Holberg et al. 2002, 2008; Sion et al. 2009; Giammichele et al. 2012; Holberg et al. 2016). Gaia DR2 has improved the completeness of these samples, recovering 130 known white dwarfs within $20 \mathrm{pc}$ and identifying 9 new candidates (Hollands et al. 2018b). It has also allowed a fairly complete census of double degenerates or white dwarfs as part of a wide binary system. Hollands et al. (2018b) have estimated the Gaia DR2 detection probability to be close to 99 per cent of all white dwarfs at $20 \mathrm{pc}$ and it is not expected to change significantly for distances up to 70-100 pc (Gentile Fusillo et al. 2019). Previous studies have attempted to assemble larger volumelimited samples of spectroscopically confirmed white dwarfs, e.g. within $40 \mathrm{pc}$ in the northern hemisphere (Limoges et al. 2015) where the authors estimate the completeness at 65$80 \%$. This needs to be reviewed in light of new Gaia DR2 catalogues of white dwarf candidates (Gentile Fusillo et al. 2019; Jiménez-Esteban et al. 2018) and our recent spectroscopic follow-up of these new candidates in Tremblay et al. (2020, hereafter Paper I).

Advantages of using volume-complete samples of white dwarfs are plentiful: for deriving the local stellar formation history and the age of Galactic structures (Tremblay et al. 2014b; Limoges et al. 2015; Isern 2019; Kilic et al. 2019; Fantin et al. 2019), studying binary evolution including mergers (Holberg et al. 2013; Toonen et al. 2017; Cheng et al. 2019; Temmink et al. 2019), statistics of evolved planetary systems (Zuckerman et al. 2010; Hollands et al. 2018a), constraining the origin of stellar magnetism (Ferrario et al. 2015; Landstreet \& Bagnulo 2019), deriving the initial-to-final-mass relation (El-Badry et al. 2018), testing white dwarf model accuracy and spectral evolution (Tremblay et al. 2019a; Genest-Beaulieu \& Bergeron 2019; Coutu et al. 2019; Ourique et al. 2019; Blouin et al. 2019; Gentile Fusillo et al. 2020; Cunningham et al. 2020), and deepening our understanding on dense matter physics including crystallisation (Blouin et al. 2019; Tremblay et al. 2019b). This work focuses on the spectroscopic volume-limited sample of white dwarfs within $40 \mathrm{pc}$ in the northern hemisphere. We use the Gaia DR2 white dwarf catalogue of Gentile Fusillo et al. (2019) as a starting point. The volume contained within the northern $40 \mathrm{pc}$ hemisphere is a factor of four larger than the all-sky $20 \mathrm{pc}$ sample of Hollands et al. (2018b), offering a significant advantage in terms of number statistics for the studies described above.

Since April 2018 we have spectroscopically observed most of the new Gaia white dwarf candidates within $40 \mathrm{pc}$ in the northern hemisphere. The spectral types and stellar parameters for more than one hundred new white dwarfs are presented in the companion Paper I. In this work, we focus on the statistics of the overall $40 \mathrm{pc}$ sample, combining spectral types from the literature and Paper I with a new photometric analysis using Gaia and Pan-STARRS data. This approach is inspired by our earlier study of Gaia DR2 white dwarfs within 20 pc (Hollands et al. 2018b). We describe the sample and its completeness in Section 2. We discuss the kinematics properties in Section 3, the sub-sample of binaries in Section 4, and discuss the space density, mass distributions, magnetism and crystallisation in Section 5. We conclude in Section 6.

\section{SAMPLE}

Our sample was obtained from a subset of the candidate white dwarf Gaia DR2 catalogue compiled by Gentile Fusillo et al. (2019). A simple cut was made to find all objects with parallaxes greater than 25 mas, i.e. $D<40 \mathrm{pc}$, resulting in 1233 objects, 1048 of which are high probability white dwarfs $\left(P_{\mathrm{WD}}>0.75\right)$. The parameter quantifying the probability of a source being a white dwarf was calculated in Gentile Fusillo et al. (2019) by creating a distribution map in HRD space of both spectroscopically confirmed Sloan Digital Sky Survey (SDSS) white dwarfs and contaminants.

Of the 1233 candidates, 587 are located in the northern hemisphere. Cross-matching with catalogues of confirmed white dwarfs from the literature (e.g., Limoges et al. 2015, Gianninas et al. 2011, Kawka \& Vennes 2012, Subasavage et al. 2017, full list in Table A1), we find that 410 of the Gaia sources correspond to white dwarfs with spectral types known before Gaia DR2. Of the remaining 177 sources that were newly identified in Gaia, 137 were observed spectroscopically and recently classified either in Paper $\mathrm{I}^{1}$ or in other recent papers (see, e.g., Landstreet \& Bagnulo 2019, 2020). 111 of them turned out to be new white dwarfs while

1 Paper I also includes updated spectral types for six northern white dwarfs. 
26 are main-sequence stars or spurious Gaia sources (see Paper I). These 26 objects and a further 37 unobserved low probability white dwarf candidates are listed in the online material (Table A2) and discussed in Section 2.3. As a consequence only three high probability white dwarf candidates specifically discussed in Section 2.2 do not currently have spectral types. Our final volume-limited Gaia sample of 521 confirmed white dwarfs and three high-probability white dwarf candidates is presented in the online material (Table A1), with the description of the data content in Table 1 . The objects are sorted by their WD J names as introduced in Gentile Fusillo et al. (2019) while column 2 uses the WD name designation for objects cross-matched with the literature and known prior to Gaia DR2. Columns 3 to 11 repeat key data from Gaia DR2 and Gentile Fusillo et al. (2019), while columns 12 to 23 report information on the atmosphere and stellar parameters as discussed in Section 2.1. Fig. 1 presents an overview in the HRD of the known and new white dwarfs within $40 \mathrm{pc}$.

We are missing local white dwarfs that were not selected by Gentile Fusillo et al. (2019). This can be because the Gaia DR2 five-parameter astrometric solution or colours are omitted or unreliable, or because the white dwarf is in an unresolved binary system and lies outside of their selection in the HRD. We have scanned the Montreal White Dwarf Database (Dufour et al. 2017) for objects within $40 \mathrm{pc}$ that are not in the full catalogue of Gentile Fusillo et al. (2019), finding a total of 22 white dwarfs presented in Table A3 that we now discuss in turn.

There are 13 white dwarfs for which a parallax value from Gaia or other sources (van Altena et al. 1995; van Leeuwen 2007; Leggett et al. 2018) confirms 40 pc membership but that were not selected in Gentile Fusillo et al. (2019) owing to missing, incomplete or unreliable Gaia data. More than half of these objects are within $20 \mathrm{pc}$ and already discussed in Hollands et al. (2018b). In most cases these missing white dwarfs are nearby $(<10 \mathrm{pc})$, have large proper motions or are close to a bright stellar companion.

There are a further six multiple stellar systems which are known to include a white dwarf but are missing from our initial sample; UZ Sex, V EG UMa, tet Hya, V DE CVn, HD 169889, and LHS 1817. These systems listed in Table A3 are all unresolved main-sequence + white dwarf binaries with complete Gaia DR2 data. They were not selected by Gentile Fusillo et al. (2019) because the main-sequence star largely dominates the Gaia photmetry. As a consequence, these white dwarfs lay outside the region where they made their cuts on the HRD. As these binary systems were gathered from various literature sources, it remains a challenge to quantify their completeness within $40 \mathrm{pc}$. We discuss these objects further in Section 4.2. We also note a small number of cool and red objects $\left(G_{\mathrm{BP}}-G_{\mathrm{RP}}>1.0\right)$ lying just above the white dwarf sequence in Fig. 1, which covers both the southern and northern hemispheres. These are unobserved low probability white dwarf candidates from Gentile Fusillo et al. (2019) and are listed in Table A2, a small number of which may be double degenerate binaries missing from our final sample.

Finally, Table A3 includes three white dwarfs with no parallax from any source, but that are possible $40 \mathrm{pc}$ members based on previously published spectroscopic or estimated photometric distances. We do not include any of the missing white dwarfs listed in Table A3 in the following statistical analysis to ensure homogeneity of the data. We conclude that our $40 \mathrm{pc}$ sample of Table A1 is at most 96 per cent complete, but that the final completeness is very likely to be close to that value (Gentile Fusillo et al. 2019).

We consider 33 additional northern objects from the catalogue of Gentile Fusillo et al. (2019) that are within $2 \sigma$ of a parallax of 25 mas separately in Table A4. For simplicity the table merges together 17 confirmed white dwarfs (nine of which were observed in Paper I), two main-sequence contaminants and 14 white dwarf candidates that have not been observed, only three of which are high probability white dwarf candidates. A few of these white dwarfs may turn out to be $40 \mathrm{pc}$ members with the improved astrometry from Gaia DR3, but to ensure that our statistics represent a volumelimited sample, we do not include any of these additional objects in our main analysis below.

\subsection{Spectral types and atmospheric parameters}

For each $40 \mathrm{pc}$ white dwarf we have gathered the spectral type from the literature, with the references given in Table A1. A breakdown of the spectral types can be seen in Table 2 (see, e.g., Sion et al. 1983 for spectral type definitions). In the majority of cases, the spectral type is sufficient to conclude that the dominant constituent of the atmosphere is either hydrogen or helium, e.g. from the presence or absence of hydrogen Balmer lines. We do not have evidence of any carbon-dominated atmosphere white dwarf in the northern $40 \mathrm{pc}$ sample, although three magnetic white dwarfs with unknown absorption bands (spectral type DX or DXP) have an ambiguous atmospheric composition. Below effective temperatures $\left(T_{\text {eff }}\right)$ of $5000 \mathrm{~K}$, the vast majority of white dwarfs are of featureless DC spectral type and it is not straightforward to determine the atmospheric composition (Blouin et al. 2019). A few of these cool objects are of metal-rich DZ or DZA spectral types, allowing to constrain the atmospheric composition based on the effect of helium or hydrogen broadening on the metal lines.

The classification into different spectral subtypes is sensitive to the signal-to-noise ratio $(\mathrm{S} / \mathrm{N})$ of the spectroscopic observations. In particular, the detection of weak metal or carbon lines as well as weak magnetic fields $(B \lesssim 500 \mathrm{kG})$ is only possible at sufficiently high $\mathrm{S} / \mathrm{N}$. This is also the case for cool hydrogen atmosphere DA white dwarfs with only weak $\mathrm{H} \alpha$ lines at $T_{\mathrm{eff}} \approx 5000 \mathrm{~K}$. A visual inspection of the published spectroscopic studies of local white dwarfs (Giammichele et al. 2012; Limoges et al. 2015; Gentile Fusillo et al. 2019; Tremblay et al. 2020) indeed reveals variations in S/N. Furthermore, when available we have updated our spectral types based on dedicated high-resolution observations or spectropolarimetry, which have observed metal lines or magnetic fields not seen in lower resolution observations. Many of these high-resolution surveys have favoured close (20 pc) and brighter white dwarfs (see, e.g., Zuckerman et al. 2003; Landstreet \& Bagnulo 2019).

The distribution of spectral types as a function of distance is shown in Fig. 2. The fractions of magnetic, DZ, and DQ white dwarfs within $20 \mathrm{pc}<d<40 \mathrm{pc}$ are all within $2 \sigma$ of those found for the $20 \mathrm{pc}$ sample, implying that biases due to $\mathrm{S} / \mathrm{N}$ of the observations are only marginally significant given the small size of the $20 \mathrm{pc}$ sample. This nevertheless 
Table 1. Format of the online catalogue which can be accessed at this link.

\begin{tabular}{llll}
\hline Index & Column Name & Units & Description \\
\hline 1 & WDJ Name & - & WDJ + J2000 ra (hh mm ss.ss) + dec (dd mm ss.s), equinox and epoch 2000 \\
2 & WD Name & - & WD Name (for objects known before Gaia only) \\
3 & Source ID & - & Gaia DR2 source identifier \\
4 & Parallax & mas & Parallax of the source \\
5 & Parallax_Error & mas & Standard error of parallax \\
6 & RA & deg & Right ascension (J2015.5) \\
7 & RA_Error & mas & Standard error of right ascension \\
8 & DEC & deg & Declination $(J 2015.5)$ \\
9 & DEC_Error & mas & Standard error in declination \\
10 & appG & mag & Apparent $G$ magnitude \\
11 & bp_rp & mag & G BP - GRP colour index \\
12 & SpT & - & Spectral Type \\
13 & Comp & - & Composition (H for hydrogen dominated or He for helium dominated) \\
14 & Gaia Teff & $\mathrm{K}$ & Adopted Gaia effective temperature \\
15 & Gaia Teff Error & $\mathrm{K}$ & Standard error of adopted Gaia effective temperature \\
16 & Gaia log(g) & {$\left[\mathrm{cm} / \mathrm{s}^{2}\right]$} & Adopted Gaia surface gravity \\
17 & Gaia log(g) Error & {$\left[\mathrm{cm} / \mathrm{s}^{2}\right]$} & Standard error on adopted Gaia surface gravity \\
18 & Pan-STARRS Teff & $\mathrm{K}$ & Pan-STARRS effective temperature \\
19 & Pan-STARRS Teff Error & $\mathrm{K}$ & Standard error on Pan-STARRS effective temperature \\
20 & Pan-STARRS log(g) & {$\left[\mathrm{cm} / \mathrm{s}^{2}\right]$} & Pan-STARRS surface gravity \\
21 & Pan-STARRS log(g) Error & {$\left[\mathrm{cm} / \mathrm{s}^{2}\right]$} & Standard error on Pan-STARRS surface gravity \\
22 & Bibcode & - & Reference paper for spectral type \\
23 & Comment & - & Additional comment \\
\hline
\end{tabular}

Table 2. Breakdown of the identified spectral types of the northern 40 pc sample.

\begin{tabular}{lll}
\hline Spectral Type & Total Number & Model Composition \\
\hline DA & 282 & pure-H $($ except He for two He-rich DA) \\
DAe & 1 & pure-H \\
DAH or DAP & 23 & pure-H \\
DAZ & 21 & pure-H \\
DAZH & 1 & pure-H \\
DB & 1 & $\mathrm{H} / \mathrm{He}=10^{-5}$ \\
DB+dM & 1 & $\mathrm{H} / \mathrm{He}=10^{-5}$ \\
DBA & 2 & $\mathrm{H} / \mathrm{He}=10^{-5}$ \\
DBAZ & 1 & $\mathrm{H} / \mathrm{He}=10^{-5}$ \\
DBP & 1 & $\mathrm{H} / \mathrm{He}=10^{-5}$ \\
DC & 145 & $\mathrm{H} / \mathrm{He}=10^{-5}$, pure-He below $7000 \mathrm{~K}$, assumed pure-H below $5000 \mathrm{~K}$ \\
DCP & 2 & $\mathrm{H} / \mathrm{He}=10^{-5}$, pure-He below $7000 \mathrm{~K}$ \\
DQ & 14 & $\mathrm{H} / \mathrm{He}=10^{-5}$, pure-He below $7000 \mathrm{~K}$ \\
DQP & 1 & $\mathrm{H} / \mathrm{He}=10^{-5}$, pure-He below $7000 \mathrm{~K}$ \\
DQpecP & 1 & $\mathrm{H} / \mathrm{He}=10^{-5}$, pure-He below $7000 \mathrm{~K}$ \\
DQZA & 1 & $\mathrm{H} / \mathrm{He}=10^{-5}$, pure-He below $7000 \mathrm{~K}$ \\
DZ & 13 & $\mathrm{H} / \mathrm{He}=10^{-5}$, pure-He below $7000 \mathrm{~K}$ \\
DZA & 4 & $\mathrm{H} / \mathrm{He}=10^{-5}$, pure-He below $7000 \mathrm{~K}$ (except H for one H-rich DZA) \\
DZH or DZP & 3 & $\mathrm{H} / \mathrm{He}=10^{-5}$, pure-He below $7000 \mathrm{~K}$ \\
DX or DXP & 3 & assumed $\mathrm{H} /$ He$=10^{-5}$, pure-He below $7000 \mathrm{~K}$ \\
Unknown & 3 & assumed pure-H \\
\hline
\end{tabular}

suggests that deeper observations of the $40 \mathrm{pc}$ sample could lead to an increase of a factor of about two in the number of detected subtypes, and one should be cautious in the determination of the absolute fraction of magnetic, DZ, and DQ white dwarfs using the $40 \mathrm{pc}$ sample.

While atmospheric parameters derived from fits to optical spectroscopy can be gathered in the literature for the warmest objects in the sample, here we take advantage of the high-precision broadband photometry and astrometry from Gaia to derive a homogeneous set of high-precision atmospheric parameters. It should be noted that a systematic offset at the few percent level has been observed between the
Gaia and spectroscopic temperature scales, both for warm DA or DB white dwarfs as well as cool DA stars (Tremblay et al. 2019a, 2020; Genest-Beaulieu \& Bergeron 2019), hence one should be cautious about the absolute temperature and mass scales.

We used pure-hydrogen (Tremblay et al. 2011), purehelium (Bergeron et al. 2011), and mixed model atmospheres (Tremblay et al. 2014a) to calculate $T_{\text {eff }}$ and surface gravity $(\log g)$ based on Gaia $G, G_{\mathrm{BP}}$ and $G_{\mathrm{RP}}$ photometry as well as parallax for each white dwarf in the sample. The fitting method is the same as that described in Gentile Fusillo et al. (2019). In brief, we rely on the Ly $\alpha$ broadening of 


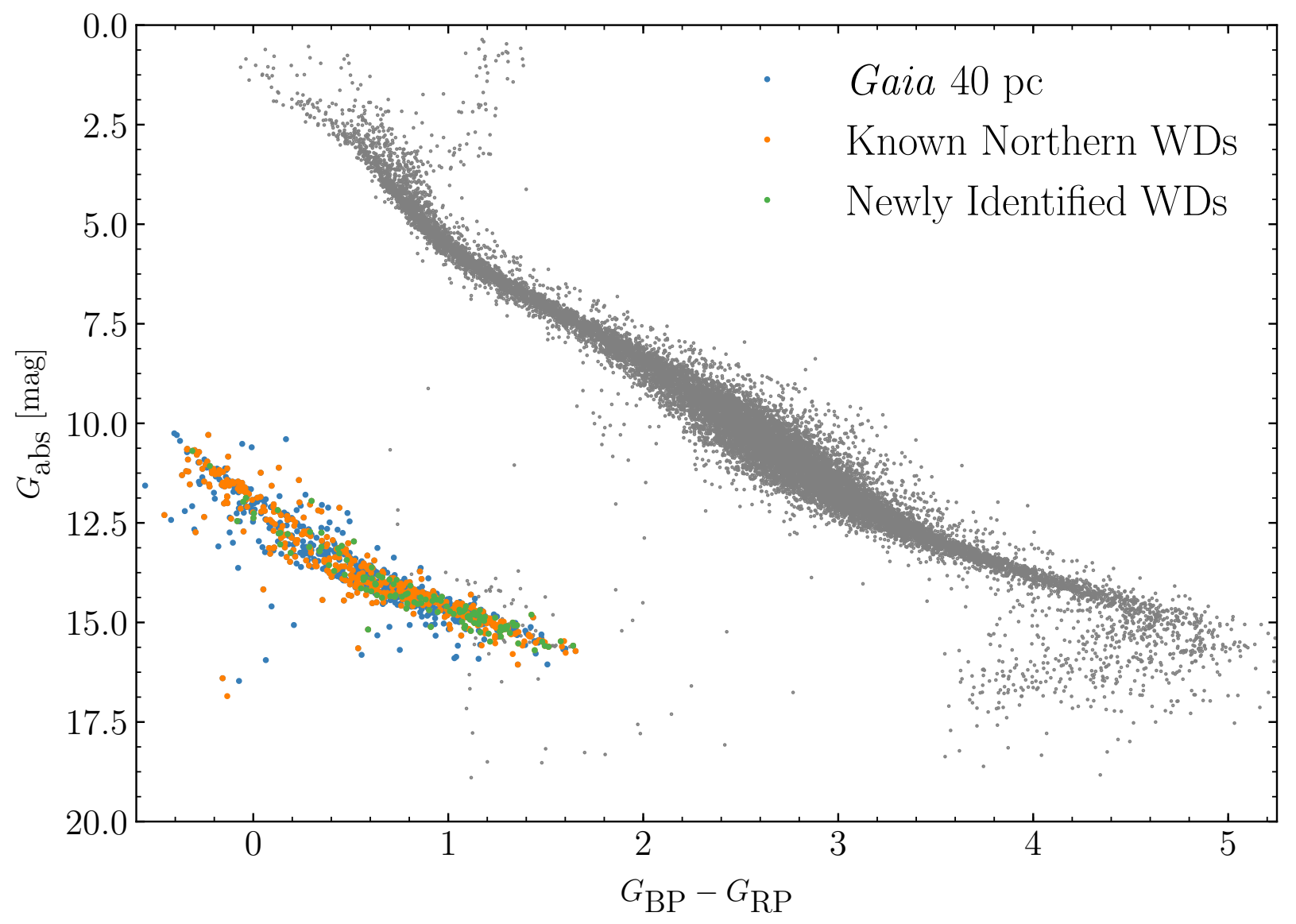

Figure 1. Hertzsprung-Russell diagram of the 40 pc population detected by Gaia. The high-probability Gaia 40 pc white dwarf sample from Gentile Fusillo et al. (2019), both in the northern and southern hemispheres, is shown in blue, with the pre-Gaia northern white dwarfs (DEC > 0) shown in orange, and the newly confirmed white dwarfs from Paper I in green. For reference, a Gaia 40 pc sample is shown in grey, cleaned using the same methodology as Gaia Collaboration et al. (2018b)

Kowalski \& Saumon (2006) and the mass-radius relation of Fontaine et al. (2001) for thick (H-atmospheres) or thin (Heatmospheres) hydrogen envelopes and C/O-cores. The only differences are that we have completely neglected reddening, which is justified for the $40 \mathrm{pc}$ sample, and we have added the option of mixed $\mathrm{He} / \mathrm{H}$ atmospheres.

Given that we have now secured a breakdown of the spectral types for all but three objects in the sample, we adopt the atmospheric parameters based on the inferred composition. This is an improvement with respect to Gentile Fusillo et al. (2019) who provided both H- and Heatmosphere options. Assignations of the different spectral types to an atmospheric composition are given in Table 2.

We have taken advantage of the essentially complete northern hemisphere coverage of the Pan-STARRS survey (Chambers et al. 2016) to derive an independent set of atmospheric parameters based on grizy photometry and Gaia parallaxes. It was demonstrated in Gentile Fusillo et al. (2019) that Pan-STARRS and Gaia photometry are in good agreement for warm DA white dwarfs, and here we explore this further with the cool white dwarfs within 40 pc. Fig. 3 demonstrates that $\log g$ and $T_{\text {eff }}$ values are generally within agreement at the few percent level and within combined error bars. Most of the outliers are in crowded areas of the sky or close to a bright companion (see Paper I). Below $T_{\text {eff }} \approx 5000 \mathrm{~K}$, Gaia colours are systematically redder than Pan-STARRS colours (and predicted white dwarf cooling sequences, see, e.g., Bergeron et al. 2019), resulting in systematically lower temperatures and surface gravities. It is unclear if this is a calibration effect or inaccurate physics influencing the Gaia and Pan-STARRS bandpasses differently.

For most local white dwarfs, the Gaia passbands are broad enough that the colours are not significantly impacted by average metal pollution or magnetic fields. Furthermore, the large majority of the objects are cool enough that metal line UV blanketing is expected to be negligible. Fig. 3 demonstrates that we obtain similar atmospheric pa- 


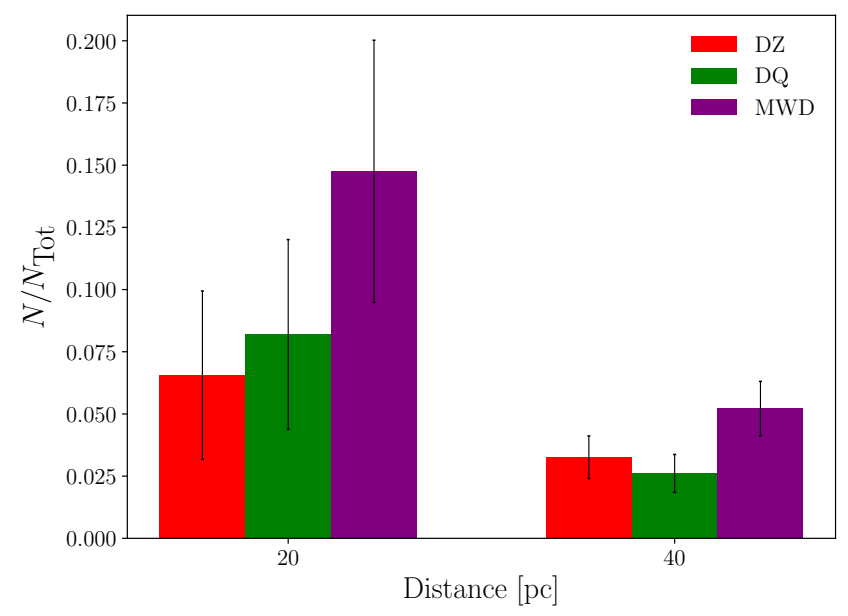

Figure 2. Distribution of spectral types as a function of distance with DZs shown in red, DQs in green, and magnetic white dwarfs in purple. The first bins correspond to the $20 \mathrm{pc}$ sample and the second bins to the $20 \mathrm{pc}<d<40 \mathrm{pc}$ sample.

rameters with the much narrower Pan-STARRS filters, apart from DZ white dwarfs which appear to be more scattered. Overall, we conclude that using pure-H, pure-He and mixed $\mathrm{H} / \mathrm{He}$ models is not a major concern for our analysis.

There are two cool DQ and DQpec with very broad and deep absorption bands. There are also four ultra-cool DC white dwarfs with significant collision-induced absorption (CIA). Those do not have meaningful Gaia or PanSTARRS atmospheric parameters and are flagged with a $\mathrm{He}+\mathrm{CIA}$ composition in Table A1 where appropriate.

Gentile Fusillo et al. (2019) and Bergeron et al. (2019) have shown that cool helium-rich white dwarfs with $7000 \mathrm{~K}$ $\lesssim T_{\text {eff }} \lesssim 11000 \mathrm{~K}$ have larger masses than their warmer DB/DBA progenitors when fitted with pure helium models. This is inconsistent with an expected stellar evolution at constant mass. Bergeron et al. (2019) have demonstrated that by adding trace hydrogen in the helium-rich atmospheres, the predicted masses are lower and in much better agreement with the expectation of evolution models. The presence of hydrogen is also consistent with DC and DZ white dwarfs being the cooler counterparts of DB/DBA white dwarfs, for which of the order of 50 per cent of objects have detectable hydrogen $\left(\mathrm{H} / \mathrm{He}>10^{-7}\right.$ in number $)$ with a median value of $\mathrm{H} / \mathrm{He} \approx 10^{-5}$ (Rolland et al. 2018). Such trace hydrogen abundance is not detectable in helium-rich DC or DZ white dwarfs cooler than about $11000 \mathrm{~K}$ for typical low- or medium-resolution observations (Rolland et al. 2018) but is needed for the large majority of these cool objects to fit the predicted cooling tracks. As a consequence, we have adopted helium-rich model atmospheres with $\mathrm{H} / \mathrm{He}=$ $10^{-5}$ for all helium-rich spectral types warmer than $7000 \mathrm{~K}$.

Below $T_{\text {eff }}=7000 \mathrm{~K}$, our mixed model atmospheres (Tremblay et al. 2014a) predict a blue hook in Gaia colours due to CIA opacities, which is not observed for the vast majority of white dwarfs (Gentile Fusillo et al. 2020). This blue hook is also not predicted in more recent mixed $\mathrm{H} / \mathrm{He}$ model atmospheres discussed in Blouin et al. (2019). As a consequence, we use instead pure-helium solutions for all helium-rich objects below $7000 \mathrm{~K}$, where the bifurcation to high masses is not observed. Finally, most objects below $5000 \mathrm{~K}$ are DC white dwarfs and it is not possible to determine the atmospheric composition based on spectroscopy and optical photometry alone, and challenging even with near-IR photometry (Gentile Fusillo et al. 2020). Therefore, we assign a pure-hydrogen composition for all objects below that temperature. We note that in the range $7000 \mathrm{~K} \gtrsim T_{\text {eff }} \gtrsim$ $4500 \mathrm{~K}$ our pure-H and pure-He solutions differ only by a few percent. As a consequence our $T_{\text {eff }}$ and $M$ estimates are, in principle, still robust even with an unconstrained composition.

Fig. 4 shows the $\log g$ versus $T_{\text {eff }}$ distribution, using both Gaia and Pan-STARRS data, for $40 \mathrm{pc}$ white dwarfs where six ultra-cool and DQ white dwarfs with unreliable Gaia parameters are excluded. The crystallisation sequence (Tremblay et al. 2019b) is clearly seen as a parabola with $\log g$ increasing as a function of $T_{\text {eff }}$, starting at $6000 \mathrm{~K}$ and $\log g \approx 8.1$ and increasing to $\log g \approx 8.7$ at $10000 \mathrm{~K}$. In the $100 \mathrm{pc}$ sample of Tremblay et al. (2019b) and $200 \mathrm{pc}$ sample of Cheng et al. (2019) the crystallisation sequence can be seen to extend to higher temperatures and surface gravities, but given the limited volume of the $40 \mathrm{pc}$ sample, the $\log g>8.7$ region (corresponding to $M \gtrsim 1.05 \mathrm{M}_{\odot}$ ) is underpopulated. Most white dwarfs on the crystallisation sequence are of DA spectral type, as highlighted in Tremblay et al. (2019b). We discuss the crystallisation sequence further in Section 5.5.

We observe that parameters for $\mathrm{H}$ - and He-rich atmospheres have no obvious offset when using mixed $\mathrm{He} / \mathrm{H}$ instead of pure-He models, as highlighted in Bergeron et al. (2019) and effectively correcting the so-called bifurcation problem (Gentile Fusillo et al. 2019).

For $T_{\text {eff }} \lesssim 5500 \mathrm{~K}, \log g$ values decrease with decreasing temperature as previously highlighted for the 20 pc sample in Hollands et al. (2018b). For fixed mass-radius relation, parallax and apparent magnitude, the effective temperature and surface gravity correlate because of the Stefan-Boltzmann law. This suggests that the problem could arise either from photometric colours that are predicted too blue or absolute magnitudes that are predicted too faint. A very similar pattern is seen with independent grids of models (Blouin et al. 2019), and for any atmospheric composition including pure-H, pure-He or mixed (Bergeron et al. 2019). The problem is clearly seen using either Gaia or Pan-STARRS photometry, although the issue is slightly worse with Gaia (see Fig. 3). It is not expected to be a real astrophysical effect as white dwarfs at these temperatures have cooling ages in the range $\approx 5-10 \mathrm{Gyr}$. Stellar population models predict a constant mean mass for white dwarfs with cooling ages smaller than about 10 Gyr (Tremblay et al. 2016). Therefore, the lower than average $\log g$ (or $T_{\text {eff }}$ ) values are more likely to be explained from an issue with the model atmospheres. The problem appears for white dwarfs with a vast range of cooling ages, hence this should be taken into account properly in order to extract meaningful stellar formation histories when transforming white dwarf parameters to initial stellar parameters (Tremblay et al. 2014b).

\section{$2.2 \quad$ Missing spectral types}

There are three objects for which ground-based spectroscopic observations are challenging and currently not avail- 


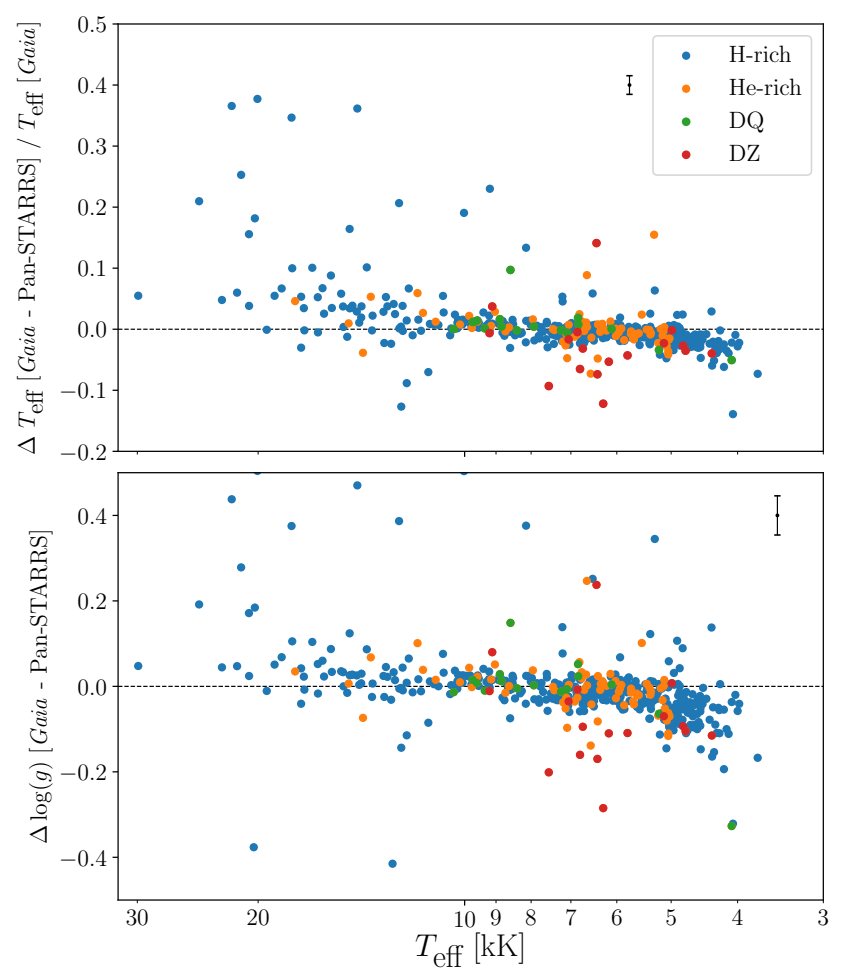

Figure 3. Difference between the measured Gaia and PanSTARRS atmospheric parameters; fractional $T_{\text {eff }}$ difference is shown in the top panel and absolute $\log g$ difference in the bottom. Both are shown as a function of $T_{\text {eff }}$. The average formal errors on the difference are shown in the top right of both panels.

able in the literature. All three are listed in Table A1 but without a spectral type.

J050600.41+590326.89 is a high proper-motion (346.95 mas/yr) Gaia source that was blended with a distant background star at the time of our observations in $\mathrm{Pa}$ per I. The object is extremely faint $(G=19.65)$ and blue $\left(G_{\mathrm{BP}}-G_{\mathrm{RP}}=-0.1335\right)$. Due to the faintness, the Gaia measurements have high astrometric excess noise, suggesting we must be careful about its status, either as a rare ultra-cool white dwarf or as a non-degenerate source. For this reason the atmospheric parameters are omitted in Table A1.

$\mathbf{J 0 5 5 6 0 2 . 0 1 + 1 3 5 4 4 6 . 7 1}$ is a high proper-motion (608.06 mas/yr) Gaia source that was close to a distant background star at the time of our observations in Paper I. The white dwarf candidate is itself a wide companion to the bright star HD 39881 of spectral class G8 at $47.7^{\prime \prime}$ separation. The presence of a wide companion at the same distance and proper motion makes the identification as a white dwarf fairly secure.

$\mathbf{J 1 1 0 1 4 3 . 0 4 + 1 7 2 1 3 9 . 3 9}$ is in the glare (17" separation) of the background F-type main-sequence star HD 95518 with $G=8.37$.

\subsection{Non-white dwarfs}

Table A2 lists 26 objects confirmed as main-sequence stars and a further 37 unobserved low probability white dwarf candidates from the initial sample of Gentile Fusillo et al.

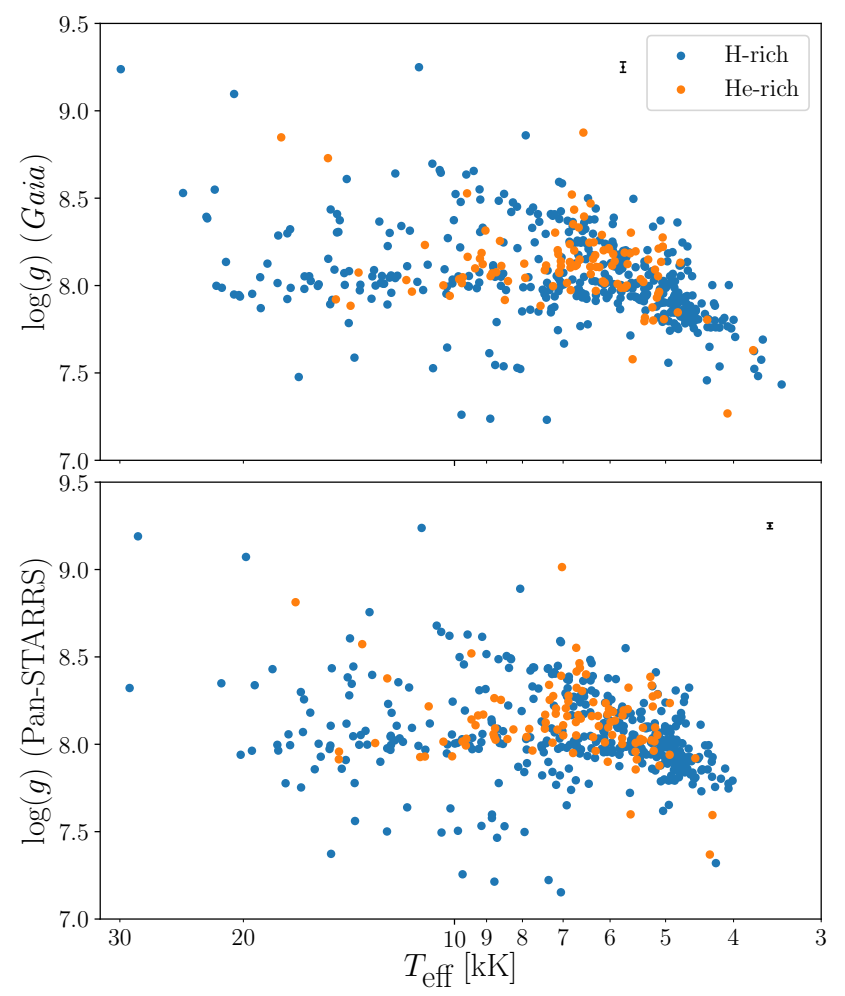

Figure 4. Distribution of $\log g$ vs $T_{\text {eff }}$ for Gaia DR2 (top panel) and Pan-STARRS (bottom) photometry. We rely on Gaia DR2 astrometry in both cases. H-rich objects are shown in blue, whilst He-rich are shown in orange. The average errors are shown in the top right of both panels. Reliable Gaia and Pan-STARRS atmospheric parameters are available for 517 and 492 objects in the sample, respectively.

(2019). None of the objects they selected within the northern 40 pc sample were known as non-white dwarfs in the literature before Gaia. All 26 main-sequence stars were therefore identified as part of our recent spectroscopic follow-up of white dwarf candidates in Paper I. Seven out of the 26 Gaia sources found to be main-sequence stars were high probability white dwarf candidates $\left(P_{\mathrm{WD}}>0.75\right)$. This is a relatively small fraction of the final white dwarf sample $(1.3$ per cent), and therefore it largely confirms the cleanness of high-probability white dwarf samples selected from Gentile Fusillo et al. (2019). Paper I concludes that a problem with astrometry is the most likely explanation for the spurious low luminosity of these stellar sources that are located well within the white dwarf cooling track according to the Gaia DR2 HRD. In all but one case ${ }^{2}$ the Pan-STARRS colours do agree with Gaia colours.

The 40 pc sample of Gentile Fusillo et al. (2019) contained 66 low probability white dwarf candidates in the northern hemisphere. Among those, eight objects are white dwarfs that were known before Gaia. A further 21 sources were recently observed in Paper I but only 2 objects turned out to be white dwarfs. There are 37 objects that have

\footnotetext{
2 In one case the Gaia source does not correspond to a real object
} on the sky. 


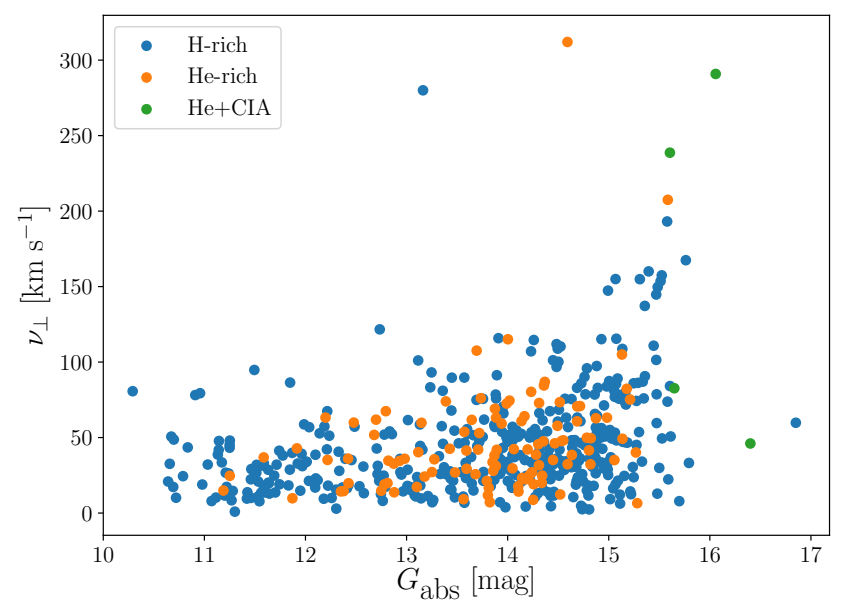

Figure 5. Tangential velocities of the sample as a function of $G_{\text {abs }}$ magnitude. H-rich objects are shown in blue, whilst Herich objects are shown in orange. Four ultracool white dwarfs are shown in green.

not been spectroscopically observed, and among them there could be a few white dwarfs. We have attempted to use kinematics or infrared colours to reveal their nature but found that any cut on only one of these quantities would also eliminate known white dwarfs. Gaia DR3 is expected to help in defining a cleaner and more complete 40 pc sample.

\section{KINEMATICS}

We can calculate tangential velocities, $v_{\perp}$, for our sample with Gaia data alone using

$v_{\perp}=4.7405 \sqrt{\mu_{R A}^{2}+\mu_{D e c}^{2}} / \varpi$

where $\mu_{R a / D e c}$ are the right ascension/declination components of the proper motion in mas $\mathrm{yr}^{-1}$, and $\varpi$ is the parallax of the white dwarf in mas. Fig. 5 shows $v_{\perp}$ as a function of $G_{\text {abs }}$, which is a proxy for cooling age. We expect that throughout their full evolution on the main-sequence and white dwarf cooling sequence, these stars will be subject to kinematic heating by the Galactic potential, with increasing $v_{\perp}$ as a function of total age (Seabroke \& Gilmore 2007). While white dwarf cooling age is only a fraction of the total age, larger cooling ages lead to, on average, larger total ages, and therefore cooling age should also correlate with kinematics, as observed in Fig. 5. In a future study we will consider total ages, which depend more critically on the absolute precision of our white dwarf masses.

Fig. 5 also reveals that the vast majority of $40 \mathrm{pc}$ white dwarfs are consistent with Galactic disc kinematics. Only four objects are clear outliers in the diagram:

$\mathbf{J 2 2 2 5 4 7 . 0 7 + 6 3 5 7 2 7 . 3 7 ,} v_{\perp}=312 \mathrm{~km} \mathrm{~s}^{-1}$, is a white dwarf of spectral type DC discovered by Gaia (Tremblay et al. 2020) and previously identified as a halo white dwarf candidate in Kilic et al. (2019). Our low surface gravity ( $\log g$ $=7.80 \pm 0.03)$ suggests a large total age in agreement with halo membership.

$\mathbf{J 1 1 0 2 1 7 . 5 2 + 4 1 1 3 2 1 . 1 8 ,} v_{\perp}=291 \mathrm{~km} \mathrm{~s}^{-1}$, is an ultracool DC white dwarf first discovered by SDSS and was suggested to be an old halo object with a cooling age of 11 Gyr by Hall et al. (2008). Because of the strong CIA absorption in the optical, it is not possible to constrain the stellar mass with Gaia or Pan-STARRS data alone.

$\mathbf{J 1 7 4 9 5 0 . 1 5 + 8 2 4 6 2 6 . 0 6}, v_{\perp}=280 \mathrm{~km} \mathrm{~s}^{-1}$, is a known 20 pc DA white dwarf (WD 1756+827) first suggested to be halo candidate by Fuchs \& Jahreiß (1998) and discussed further in Hollands et al. (2018b) and Kilic et al. (2019).

$\mathbf{J 0 3 4 6 4 6 . 5 2 + 2 4 5 6 0 2 . 6 7 ,} v_{\perp}=239 \mathrm{~km} \mathrm{~s}^{-1}$, is another ultracool DC white dwarf which was previously known for its high proper-motion and halo membership (WD 0343+247; Hambly et al. 1997; Kilic et al. 2019).

\section{BINARITY}

\subsection{Wide binaries}

To search for wide binaries within our sample, both white dwarf + main-sequence (WD+MS) and double white dwarfs (WD+WD), we employ the same technique as Hollands et al. (2018b). In brief, we perform a cylindrical search around each white dwarf in our sample to a projected separation $\left(D_{\perp}\right)$ of $1 \mathrm{pc}$. For each object found, a cut was made in the absolute difference in radial distances $\left(\Delta D_{\|}\right)$, also at $1 \mathrm{pc}$. In addition we have used the standard quality cuts of Gaia Collaboration et al. (2018b) on astrometric excess noise and colour excess on all wide stellar companions. The remaining stars within the search volume were then checked for consistent tangential velocities $\left(\Delta v_{\perp}\right)$. Those with large separations and large tangential velocity differences that correspond to physically unbound systems for a total mass of $2 \mathrm{M}_{\odot}$ on circular orbits were rejected as being non-companions.

We find a total of 56 binary system candidates in our sample, these are shown in Fig. 6 and listed in Table A5. Those consist of $47 \mathrm{WD}+\mathrm{MS}, 1 \mathrm{WD}+\mathrm{MS}+\mathrm{MS}$ and $8 \mathrm{WD}+\mathrm{WD}$ binaries. From a similar Gaia DR2 selection, El-Badry \& Rix (2018) found $43 \mathrm{WD}+\mathrm{MS}$ and $8 \mathrm{WD}+\mathrm{WD}$ within the northern $40 \mathrm{pc}$ sample, all of which we recover. El-Badry \& Rix (2018) neglected triple systems and used slightly stricter selection rules as they were aiming at minimising contamination rather than maximising completeness in their much larger volume of $200 \mathrm{pc}$.

In comparison, Hollands et al. (2018b) found 23 wide binary systems within 20 pc. Of these, 21 are WD+MS binaries, and only two are WD+WD binaries. Extrapolating the space density from the $20 \mathrm{pc}$ sample we could expect 92 \pm 19 systems within $40 \mathrm{pc}$. However, it is found in Section 5 that the space density itself is seen to decrease with distance for all white dwarfs (2-5 per cent effect). Furthermore, Gaia resolving power is decreasing with distance and therefore 813 per cent lower numbers are expected for the $40 \mathrm{pc}$ sample (Toonen et al. 2017; Hollands et al. 2018b). Given these two effects and the relatively large error bars from number statistics, the difference in space density of wide systems including a white dwarf between the 20 and 40 pc samples is only marginally significant.

Based on binary population synthesis for the $50 \mathrm{pc}$ Gaia sample and re-scaled to our volume, Toonen et al. (2017) predict $85-150$ resolved WD+MS and $60-112$ resolved $\mathrm{WD}+\mathrm{WD}$ systems. These numbers can decrease by $15-30$ per cent if we consider the possible disruption of 


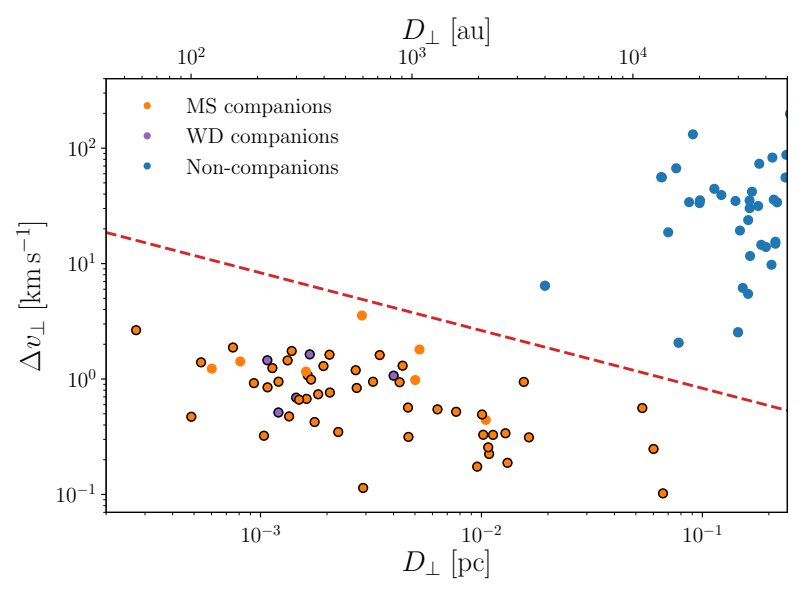

Figure 6. Tangential velocity differences as a function of projected separation for all Gaia sources within $50000 \mathrm{AU}$ of the white dwarfs in our sample. Companions also found in El-Badry \& Rix (2018) are outlined in black. The red dashed line indicates the maximum tangential velocity difference for a system of two $1 \mathrm{M}_{\odot}$ stars on circular orbits with a semi-major axis of $D_{\perp} / 2$. Our final candidates are shown in orange (WD+MS) and purple (WD+WD). Blue candidates are rejected because of their nonphysical separation in position-velocity space.

weakly bound binaries by Galactic interactions and stellar winds. The number of observed WD+MS systems is low but marginally in agreement with model predictions if a large fraction of initially formed WD+MS systems were disrupted. The issue of missing WD+WD systems is already discussed at length in Toonen et al. (2017), although the deficit is now strongly confirmed by the 40 pc sample.

El-Badry \& Rix (2018) discuss the properties of wide binaries including a white dwarf from the much larger $200 \mathrm{pc}$ sample. They find that their orbital separation distribution differs from that of wide binaries including two stars, suggesting that velocity kicks from mass loss during stellar evolution play an important role in these systems. El-Badry \& Rix (2018) notice that the deficit is enhanced for separations larger than $\approx 5000 \mathrm{au}$. While it is outside of the scope of this work to identify the physical processes in which weakly bound WD+MS and WD+WD systems are disrupted, it is hoped that our $40 \mathrm{pc}$ sample can provide further insight.

Given Gaia magnitude limit and lack of IR capabilities, our search is incomplete for substellar companions. We note the presence of at least one such confirmed system within the northern 40 pc sample, WD $1422+095$, which consists of a DA white dwarf at $33.4 \mathrm{pc}$ with a wide L4 brown dwarf companion separated by 120 AU (Becklin \& Zuckerman 1988). The brown dwarf is not detected in Gaia DR2.

Finally, at least three of our wide binaries are part of hierarchical triples. J043644.90+270951.52 is the outer companion of a spectroscopic binary of spectral type K2 (Holberg et al. 2013). J163421.55+571008.87 is in a wide orbit around an eclipsing MS-MS binary of spectral type M (Toonen et al. 2017). J170530.44+480312.36 is itself a double degenerate (see Section 4.3) with J170530.97+480310.27 as a wide companion.

\subsection{Unresolved WD+MS}

From a search of the literature, we have found six unresolved WD+MS binaries that were missing from the selection of Gentile Fusillo et al. (2019) and listed in Table A3. Hollands et al. (2018b) had identified none within the $20 \mathrm{pc}$ sample but a white dwarf companion to the M4.5V star LHS 1817 was recently discovered (Winters et al. 2020). The five other unresolved systems are beyond 20 pc. Based on binary population synthesis models that include observational selection effects, Toonen et al. (2017) predict 3-8 unresolved WD+MS binaries within our surveyed volume. The six objects found are well within that range but our sample is very likely incomplete.

The majority of confirmed unresolved WD+MS systems at all distances have a low-mass $\mathrm{M}$ dwarf companion given the relative ease of identifying binarity from optical colours and spectroscopy (Rebassa-Mansergas et al. 2016). However, recent spectroscopic follow-ups such as in Paper I have only focused on Gaia sources consistent with single and double white dwarf parameters, and therefore the $40 \mathrm{pc}$ sample has incomplete spectroscopic coverage of unresolved WD+MS candidates. This is largely because of the high level of contamination of the local sample by Gaia DR2 sources with incorrect or spurious astrometry (Gentile Fusillo et al. 2019), which has proportionally much greater impact on less crowded regions of the HRD where composite spectra corresponding to unresolved binary systems are found.

White dwarfs with more massive unresolved FGK type companions, as well as cool white dwarfs around M dwarfs, have remained elusive due to the main-sequence component usually dominating the optical flux (Holberg et al. 2013). Parsons et al. (2016) and Rebassa-Mansergas et al. (2017) identify more than a thousand WD+FGK candidates at all distances from their UV excess flux that they interpret as the likely presence of a white dwarf companion. However, the presence of a white dwarf has not been confirmed in any of their candidates within $40 \mathrm{pc}$. Follow up observations with the Hubble Space Telescope (HST) has confirmed DA white dwarfs within 8 out of 9 of their systems outside 40 pc, however. Furthermore, this technique is only sensitive to white dwarfs warm enough to have detectable UV flux. Nevertheless, these searches could be updated in light of the newer Gaia DR2 catalogue coupled with GALEX.

We note that our full sample of Table A1 contains only one spectral type $(\mathrm{DB}+\mathrm{dM})$ indicating an unresolved WD+MS system, J133601.82+482846.25, located at 36.9 pc. However, the system is resolved with Gaia DR2, and therefore classified as a wide binary system (Section 4.1).

Finally, in order to detect cool unresolved stellar companions, we have cross-matched our sample with 2MASS and WISE photometry, resulting in 481 and 429 matches, respectively (Gentile Fusillo et al. 2020). Given typical error bars of $0.05-0.10 \mathrm{mag}$ on near-IR and IR magnitudes, we have only considered strong outliers (>0.4 mag) in $J H K$ and $W 1$-band absolute magnitudes compared to the median value at a given $G_{\mathrm{BP}}-G_{\mathrm{RP}}$ colour. This resulted in seven candidates with a strong near-IR and IR excess. All of them are resolved wide systems in Gaia and discussed in Section 4.1 but are only partially resolved or unresolved in 2MASS and WISE. Therefore, this search has not resulted in any additional unresolved WD+MS systems. 


\subsection{Unresolved double white dwarfs}

Twenty-six suspected or known double degenerates and lowmass white dwarfs are identified in Table 3 (see also comments in Table A1). We discuss them in turn in this section.

We initially flagged all objects for which Gaia $\log g<$ 7.72 or the difference between the published spectroscopic and photometric $\log g$ values is greater than 0.5 dex. We have added J094846.64+242125.88 which does not fit the selection but is mentioned as a double DA+DAH in the literature (Liebert et al. 1993). The published spectroscopic $\log g$ values in Table 3 as well as our photometric Gaia estimates are under the assumption of a single white dwarf and for illustrative purpose only. A low photometric $\log g$ under this assumption either suggests a low-mass white dwarf $\left(M \lesssim 0.45 \mathrm{M}_{\odot}\right)$ formed through binary evolution and with an unseen companion, or that two white dwarfs with normal $\log g$ values instead contribute to the total flux. We have reviewed all individual cases and removed those for which a spurious spectroscopic mass, e.g. because of magnetic fields or low $\mathrm{S} / \mathrm{N}$, is the most likely explanation. Very cool white dwarfs $\left(T_{\text {eff }}<4500 \mathrm{~K}\right)$ have a low mass problem (see Fig. 4), hence we have removed all candidates below this temperature. Our list of double degenerate candidates is therefore incomplete, both because of the initial selection of Gentile Fusillo et al. (2019) which may have missed some unusually high luminosity (low-mass) sources and our secondary selection based on white dwarf parameters. However, the large majority of the objects in Table 3 are strong double dwarf candidates with no obvious alternative explanation, or already confirmed. Their mean photometric $\log g$ is 7.46 , well below the average of $\log g \approx 8.0$ for the entire sample.

Only two candidates are within $20 \mathrm{pc}$ and were previously discussed in Hollands et al. (2018b). The increase in the number of double degenerate candidates is consistent with the increase in volume, given the low number statistics of the $20 \mathrm{pc}$ sample. Furthermore, only two of the candidates are from newly identified Gaia white dwarfs (J023117.04+285939.88 and J192359.24+214103.62).

Seven objects are already confirmed multiple systems. There is a quadruple system (J010349.92+050430.57) consisting of a close double degenerate (1.2 or $6.4 \mathrm{hr}$ period) with HD 6101, a wide dK3+dK8 resolved pair with $0.5^{\prime \prime}$ separation (Maxted et al. 2000b; Toonen et al. 2017). The main sequence pair appears to have a disrupted and incorrect Gaia DR2 parallax measurement, hence the system is not part of our sample of wide binaries including a white dwarf (Section 4.1). Our only triple WD system is the previously known J170530.44+480312.36, which consists of a double degenerate with a $0.15 \mathrm{hr}$ period (Maxted et al. 2000a) and a wide white dwarf companion (see Section 4.1). Furthermore, five of the double degenerates have been confirmed in the literature, either from radial velocity variations, double cores in Balmer lines at high-resolution or a composite spectrum. This includes the low-mass white dwarf J102459.83+044610.50 with radial variations over a $1.16 \mathrm{hr}$ orbital period (Brown et al. 2011).

At least eight of our double degenerate candidates have been discussed as such in the literature, including the two objects within $20 \mathrm{pc}$. However, 10 other objects have no explicit identification as double degenerate candidates in the literature. Two of these are newly discovered white dwarfs from Gaia while eight objects had no reliable prior parallax measurements that would have been necessary to flag them as double degenerate candidates.

Of the only two confirmed low-mass white dwarfs, J094639.07+435452.24 does not have a confirmed companion although it likely formed through binary evolution (Brown et al. 2011). Both low mass white dwarfs have $\log g$ $=7.50-7.70$, based on either the photometric or spectroscopic technique, corresponding to $M=0.35-0.45 \mathrm{M}_{\odot}$. We do not have evidence of any extremely low-mass white dwarf (Kawka et al. 2020) in our sample. However, some of the double degenerate candidates described above could also harbour a low-mass white dwarf $(\log g=6.88-7.72$ according to photometric values in Table 3), especially in those cases where no spectroscopic $\log g$ value is available to confirm that the photometry is over-luminous compared to model predictions.

The binary population synthesis models of Toonen et al. (2017) predict 5-33 unresolved WD+WD binaries within our surveyed volume, well within the range observed. We hope that our improved number statistics will help to further constrain binary population synthesis models, for which one of main source of uncertainty comes from the physics of the common-envelope phase.

\section{DISCUSSION}

\subsection{Space density}

The local space density of white dwarfs has historically been estimated using the 13 or $20 \mathrm{pc}$ volume-complete censuses (Sion et al. 2009; Giammichele et al. 2012; Holberg et al. 2016; Hollands et al. 2018b) or larger samples corrected for completeness and Galactic structure effects (see, e.g., Munn et al. 2017; Jiménez-Esteban et al. 2018). Based on the Gaia DR2 20 pc sample and a careful consideration of its distance-dependent completeness, Hollands et al. (2018b) derive a space density of $(4.49 \pm 0.38) \times 10^{-3} \mathrm{pc}^{-3}$. That estimate is considerably larger than the value of $(4.15 \pm 0.35) \times$ $10^{-3} \mathrm{pc}^{-3}$ found just by accounting for the 139 white dwarfs or double degenerates detected in Gaia DR2 in the same volume. They established that Gaia DR2 misses known objects at short distances, but is close to complete for white dwarfs near $20 \mathrm{pc}$.

Gentile Fusillo et al. (2019) found that Galactic structure effects, namely the density scale height, become increasingly significant for distances beyond $\approx 20$ pc. They determined that the $100 \mathrm{pc}$ white dwarf sample is best fit with an age-averaged density scale height of $\approx 250 \mathrm{pc}$, assuming that Gaia DR2 completeness does not change significantly within that distance (see also Torres et al. 2019). While the 40 pc sample is closer to the Galactic disc, selecting only the northern hemisphere amplifies Galactic effects. The northern sample favours Galactic latitudes that are further away from the Galactic plane than the southern hemisphere. Assuming a density scale height of $250 \mathrm{pc}$, this results in a space density 2.6 per cent lower in the northern 40 pc sample compared to the full $20 \mathrm{pc}$ sample.

The actual numbers of systems including a white dwarf detected in Gaia DR2 within $20 \mathrm{pc}$ (139) and within the northern $40 \mathrm{pc}$ sample (524) lead to space densities in agree- 
Table 3. Double Degenerates in the Gaia DR2 Northern 40 pc Sample

\begin{tabular}{|c|c|c|c|c|c|c|}
\hline WDJ name & Parallax $[\mathrm{mas}]$ & $\mathrm{SpT}$ & Gaia $\log g^{1}$ & Spectro $\log g^{1}$ & Orb. Period [day] & Ref (Binarity) \\
\hline \multicolumn{7}{|c|}{ Double degenerate component of a triple or quadruple system } \\
\hline J010349.92+050430.57 & $44.86(0.12)$ & DA & $7.52(0.01)$ & $8.17(0.05)$ & 1.2 or 6.4 & Maxted et al. (2000b) \\
\hline J170530.44+480312.36 & $25.38(0.03)$ & DA & $7.24(0.01)$ & $7.67(0.05)$ & 0.1448 & Maxted et al. (2000a) \\
\hline \multicolumn{7}{|c|}{ Known double degenerate } \\
\hline J053620.21+412955.62 & $30.99(0.04)$ & DA & $7.23(0.01)$ & $7.97(0.05)$ & - & Zuckerman et al. (2003) \\
\hline J094846.64+242125.88 & $27.37(0.07)$ & DA & $8.29(0.01)$ & $8.40(0.11)$ & - & Liebert et al. (1993) \\
\hline $\mathrm{J} 131257.90+580511.29$ & $31.32(0.24)$ & DA & $7.64(0.02)$ & $8.15(0.05)$ & - & Gentile Fusillo et al. (2018 \\
\hline J164136.61+151237.93 & $31.48(0.11)$ & DA & $7.87(0.02)$ & $8.49(0.07)$ & 1471 & Harris et al. (2013) \\
\hline \multicolumn{7}{|c|}{ Double degenerate candidate (also in literature) } \\
\hline J012924.26+102301.34 & $34.14(0.05)$ & DA & $7.54(0.01)$ & $7.88(0.06)$ & - & Zuckerman et al. (2003) \\
\hline J014511.23+313243.56 & $27.85(0.09)$ & DA & $7.61(0.02)$ & $8.12(0.05)$ & - & Bédard et al. (2017) \\
\hline $\mathrm{J} 134532.97+420043.66$ & $26.68(0.07)$ & $\mathrm{DC}$ & $7.15(0.04)$ & - & - & Limoges et al. (2015) \\
\hline J163441.85+173634.09 & $39.05(0.03)$ & DAZ & $7.26(0.01)$ & $7.79(0.05)$ & - & Toonen et al. (2017) \\
\hline $\mathrm{J} 205020.65+263040.76$ & $52.34(0.05)$ & DA & $7.27(0.01)$ & - & - & Hollands et al. (2018b) \\
\hline $\mathrm{J} 211345.93+262133.27$ & $32.34(0.32)$ & DA & $7.53(0.02)$ & $8.15(0.06)$ & - & Bergeron et al. (2001) \\
\hline $\mathrm{J} 225123.02+293944.49$ & $51.47(0.14)$ & DA & $7.71(0.01)$ & - & - & Hollands et al. (2018b) \\
\hline J232519.87+140339.61 & $42.34(0.13)$ & DA & $7.31(0.01)$ & - & - & Limoges et al. (2015) \\
\hline \multicolumn{7}{|c|}{ Double degenerate candidate (this work) } \\
\hline J000754.11+394732.18 & $29.03(0.06)$ & $\mathrm{DC}$ & $6.88(0.02)$ & - & - & - \\
\hline J002215.19+423642.15 & $29.32(0.06)$ & $\mathrm{DC}$ & $7.58(0.01)$ & - & - & - \\
\hline J020847.22+251409.97 & $25.61(0.05)$ & DA & $7.48(0.01)$ & $7.91(0.05)$ & - & - \\
\hline J023117.04+285939.88 & $38.47(0.38)$ & DA & $7.67(0.02)$ & - & - & - \\
\hline J032020.30+233331.72 & $25.48(0.19)$ & $\mathrm{DC}$ & $7.21(0.05)$ & - & - & - \\
\hline J054457.66+260300.14 & $27.68(0.10)$ & DC & $7.11(0.05)$ & & - & - \\
\hline J111536.96+003317.11 & $25.22(0.14)$ & DA & $7.56(0.04)$ & - & - & - \\
\hline J192359.24+214103.62 & $28.73(0.15)$ & DA & $7.55(0.02)$ & $8.06(0.02)$ & - & - \\
\hline $\mathrm{J} 200654.88+614310.27$ & $29.84(0.09)$ & DA & $7.35(0.02)$ & - & - & - \\
\hline $\mathrm{J} 214913.61+041550.35$ & $28.44(0.09)$ & DA & $7.40(0.04)$ & - & - & - \\
\hline \multicolumn{7}{|l|}{ Low-mass white dwarf } \\
\hline J094639.07+435452.24 & $31.29(0.04)$ & DA & $7.69(0.05)$ & $7.59(0.01)$ & - & Brown et al. (2011) \\
\hline $\mathrm{J} 102459.83+044610.50$ & $25.22(0.49)$ & DA & $7.53(0.04)$ & $7.63(0.05)$ & 1.157 & Brown et al. (2011) \\
\hline
\end{tabular}

Note: ${ }^{1}$ All photometric and spectroscopic fits are assuming a single white dwarf and are shown for illustrative purpose only.

Spectroscopic parameters are from Limoges et al. (2015) or Tremblay et al. (2020). Both studies account for 3D convective effects

(Tremblay et al. 2013).

ment within $1 \sigma$. This is largely a consequence of the relatively large error on number statistics for the much smaller $20 \mathrm{pc}$ sample. However, this does not account for the fact that the Gaia detection rate is not constant as a function of distance. Given that the pre-Gaia northern 40 pc sample was at most $\approx 80$ per cent complete (Limoges et al. 2015), it is difficult to establish a robust list of white dwarfs missing from Gaia DR2. In other words, it is not sufficient to correct only for the known missing white dwarfs from Table A3 as we also need to include objects that are missing both from earlier samples and Gaia DR2. For this reason we consider that it is premature to update the white dwarf space density with larger volume samples. More work is also needed to understand the Galactic distribution and scale height of white dwarfs as a function of mass and age.

\subsection{Mass distribution}

In Fig. 7 we show the photometric mass distribution for the full $40 \mathrm{pc}$ northern sample in blue and for $T_{\mathrm{eff}}>5000 \mathrm{~K}$ in orange. Similarly to Hollands et al. (2018b) we present the reduced distribution due to the low $T_{\text {eff }}$ regime being affected by the systematic decrease in $\log g$ (see Section 2). The mass distribution of Hollands et al. (2018b) is also shown alongside ours, with the full sample shown in green, and the reduced sample in orange. Alongside the canonical peak located at $0.6 \mathrm{M}_{\odot}$, our sample does not show any significant secondary feature in the mass distribution (Tremblay et al. 2016; Hollands et al. 2018b; Temmink et al. 2019). Both the $20 \mathrm{pc}$ and $40 \mathrm{pc}$ samples have a similar mean mass value of $0.66-0.67 \mathrm{M}_{\odot}\left(T_{\text {eff }}>5000 \mathrm{~K}\right)$. The astrophysical implications of the absolute mean mass value directly depend on the accuracy of Gaia photometric calibration (Tremblay et al. 2019a; Genest-Beaulieu \& Bergeron 2019). Nevertheless, using Pan-STARRS we find a mean mass of $0.65 \mathrm{M}_{\odot}$ for the $40 \mathrm{pc}$ sample, in good agreement with the Gaia result and in the same range as previous estimates for the local volume sample (Tremblay et al. 2016).

We find the same mean mass of $0.66 \mathrm{M}_{\odot}$ for both $\mathrm{H}$ and He-rich white dwarfs $\left(T_{\text {eff }}>5000 \mathrm{~K}\right)$. This may be a co- 

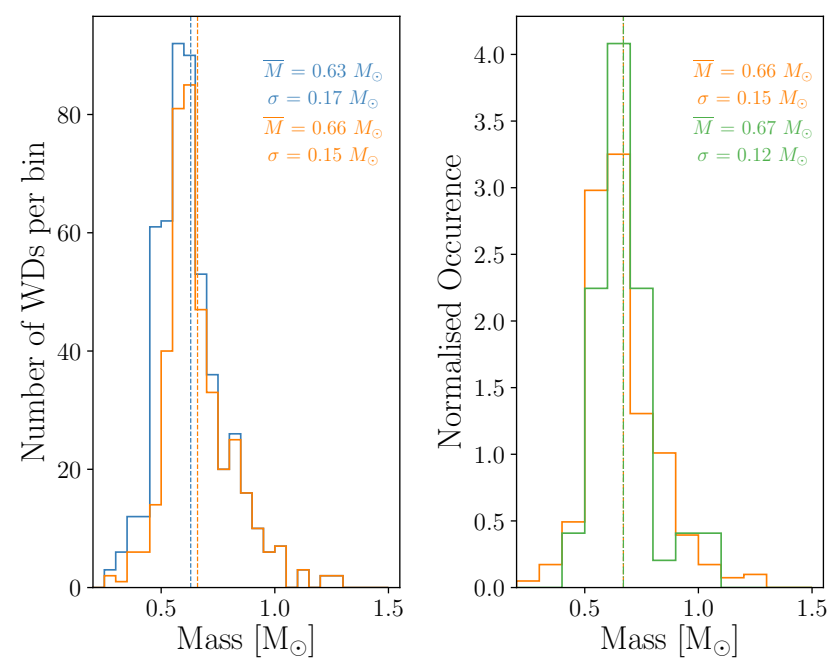

Figure 7. Left panel: Mass distribution of the northern $40 \mathrm{pc}$ sample (blue) with the reduced sample of objects with $T_{\text {eff }}>5000 \mathrm{~K}$ shown in orange $\left(\bar{M}_{40 \mathrm{pc}}=0.66 \mathrm{M}_{\odot}\right.$ and $\sigma_{40 \mathrm{pc}}=$ $\left.0.15 \mathrm{M}_{\odot}\right)$. Right panel: Similar to left, a normalised distribution with the northern $40 \mathrm{pc}$ sample $\left(T_{\text {eff }}>5000 \mathrm{~K}\right)$ in orange, and the full $20 \mathrm{pc}\left(T_{\mathrm{eff}}>5000 \mathrm{~K}\right)$ sample in green $\left(\overline{\boldsymbol{M}}_{20 \mathrm{pc}}=0.67 \mathrm{M}_{\odot}\right.$ and $\left.\sigma_{20 \mathrm{pc}}=0.12 \mathrm{M}_{\odot}.\right)$

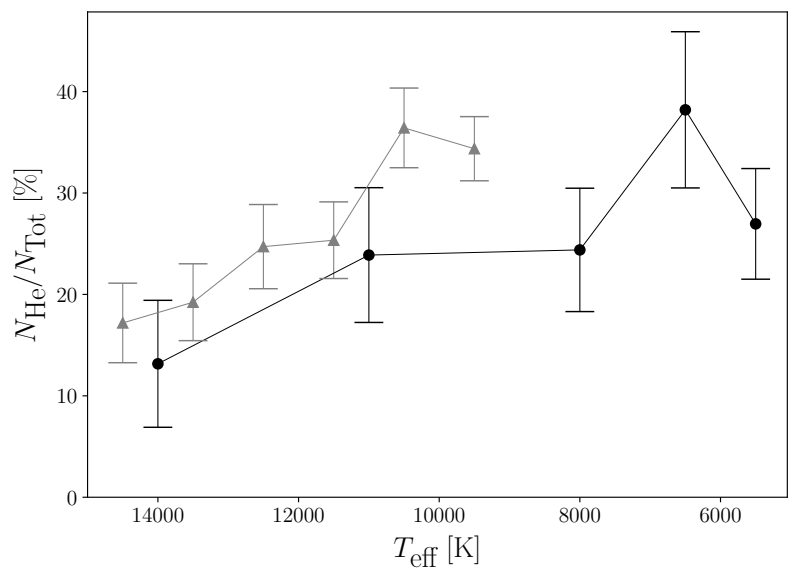

Figure 8. Percentage of He-rich atmosphere white dwarfs (DB, DC, DZ, DQ and He-rich DA) to the total as a function of effective temperature, $T_{\text {eff }}$. Data from this work is shown in black, whilst the He incidence from Cunningham et al. (2020) is shown in grey.

incidence from using He-atmosphere models with a fixed $\mathrm{H} / \mathrm{He}=10^{-5}$ trace abundance, which is not expected to fully represent the effects of hydrogen and metals in these atmospheres. Given our temperature cut, the majority of our He-rich atmospheres fall within the range where a bifurcation appears in the HRD when using pure-He models (Gentile Fusillo et al. 2019). Nevertheless, Tremblay et al. (2019a) have shown that warmer DB and DBA white dwarfs in larger magnitude limited samples have a similar mean mass to DA white dwarfs. Since DB, DBA and DA white dwarfs are thought to be the progenitors of the cool He-rich atmospheres in our sample (Cunningham et al. 2020), it is expected that they should also have the same mean mass as DA white dwarfs. This suggests that our adopted fixed trace hydrogen abundance leads to a sound astrophysical result, although the different mean masses for DC, DQ and DZ white dwarfs as described below is still a source of concern.

The 17 DQ white dwarfs have a mean mass of $0.62 \mathrm{M}_{\odot}$, which is lower than the average. Since cool DQ white dwarfs have broad optical absorption bands, the difference in mass could be explained by the fact that we use mixed $\mathrm{H} / \mathrm{He}$ atmospheres without carbon for these objects. For $20 \mathrm{DZ}$ white dwarfs we find a slightly lower mean mass $(\bar{M}=$ $\left.0.63 \mathrm{M}_{\odot}, \bar{M}_{>5000 \mathrm{~K}}=0.64 \mathrm{M}_{\odot}\right)$ than other He-atmospheres, which could be the result of our neglect of metals in model atmospheres. This effect is even more pronounced for 22 DAZ white dwarfs $\left(\bar{M}=0.58 \mathrm{M}_{\odot}, \bar{M}_{>5000 \mathrm{~K}}=0.59 \mathrm{M}_{\odot}\right)$, in which the presence of metals is not expected to contribute to any significant opacity that would lead to a systematic effect on mass determinations. The mean mass difference could be caused by selection biases in detecting metals in DA white dwarfs. Lower mass white dwarfs are more luminous for a volume-limited sample and have brighter apparent magnitudes on average, possibly facilitating metal detection. Nevertheless, the mean mass difference of $0.07 \mathrm{M}_{\odot}$ between DA and DAZ is large and unlikely to be fully explained by this bias. A Kolmogorov-Smirnov test gives a p-value of 1.9 per cent, corresponding to the likelihood that random fluctuations can explain the mean mass difference. This confirms that the mean mass difference is statistically significant but we cannot fully reject a random fluctuation. This could suggest either that planet formation occurs more frequently in lower mass stars or that planetary debris scattering onto central white dwarf occurs less frequently in remnants with higher mass progenitors (Veras et al. 2020).

\subsection{Spectral evolution}

Figure 8 shows the percentage of He-rich objects as a function of $T_{\text {eff }}$. The small number of warm white dwarfs in the $40 \mathrm{pc}$ sample does not allow to have more than 1 bin above $12000 \mathrm{~K}$. Even below that temperature, the error bars due to number statistics remain larger than the small observed fluctuations, suggesting that larger volume samples will be needed to fully address spectral evolution (MacDonald \& Vennes 1991; Bergeron et al. 2001; Tremblay \& Bergeron 2008; Blouin et al. 2019; Cunningham et al. 2020).

Below $T_{\text {eff }}=20000 \mathrm{~K}$, the only processes that have been invoked to change white dwarf surface abundances are convective mixing of the underlying helium layer with the top hydrogen layer (Rolland et al. 2018; Cunningham et al. 2020), accretion of planetary debris (Gentile Fusillo et al. 2017) or convective dredge-up of carbon (Koester et al. 1982). Only the first scenario of convective mixing is thought to result in a change of the dominant atomic species in the atmosphere, namely a transition from $\mathrm{H}$ - to He-dominated (DA to $\mathrm{DB}(\mathrm{A})$ or $\mathrm{DC}$ ). Hence, convective mixing is currently the only reasonable scenario to explain variations in Fig. 8.

Our results can be compared in Fig. 8 to the study of Cunningham et al. (2020) who employed SDSS, GALEX and Gaia photometry to study spectral evolution in the range $9000 \mathrm{~K} \leq T_{\text {eff }} \leq 20000 \mathrm{~K}$ from the strength of the Balmer jump in the $133 \mathrm{pc}$ volume-limited sample. They found a 
He-rich percentage with respect to total number of white dwarfs of $18 \pm 3$ per cent at $13000-15000 \mathrm{~K}$ and $34 \pm 3$ per cent for the bin in the range 9000-10000 K. This increase by a factor of $\approx 2$ is consistent with the picture provided by the $40 \mathrm{pc}$ sample. The He-rich fraction in the $40 \mathrm{pc}$ sample is consistently lower compared to the results found in Cunningham et al. (2020), but still agrees to within 1-2 $\sigma$ for any temperature bin given the small number statistics.

The spectral evolution for $T_{\text {eff }}<8000 \mathrm{~K}$ was also studied by Blouin et al. (2019) using a spectral type identification method similar to the one employed in this work, albeit with a different sample incomplete in volume but likely representative of the local white dwarf population. They also used different model atmospheres. Neglecting the range $T_{\text {eff }}<5000 \mathrm{~K}$ for which we did not adopt atmospheric compositions, they find a He-rich percentage of $\approx 20-25$ per cent in the range $5000-8000 \mathrm{~K}$, which is marginally lower than our average value of $30 \pm 4$ per cent.

Both studies suggest an increase and subsequent decrease in the He-rich fraction around $6500 \mathrm{~K}$, although in our case the significance is only at the $1-2 \sigma$ level. The increase can be explained by the occurrence of convective mixing in white dwarfs with relatively thick hydrogen layers $\left(\log \left(M_{\mathrm{H}} / M_{\mathrm{WD}}\right) \sim-7\right)$. But given that this process is non-reversible, there exists no obvious physical explanation for the decrease in He-rich fraction below 6500 K. Similar behaviour has been observed in previous photometric and spectroscopic studies, with an apparent deficit of He-rich objects between $\approx 6000-5000 \mathrm{~K}$ being coined the "non-DA gap" (Bergeron et al. 1997; Leggett et al. 1998; Bergeron et al. 2001). This observation was investigated by Chen \& Hansen (2012) who proposed that the non-DA gap could be explained by convective mixing in white dwarfs where the convection zone is coupled to the degenerate core. Convective coupling occurs when the base of the convection zone grows deep enough to reach the degenerate core. From evolutionary models this occurs in white dwarfs with effective temperatures of $\approx 6000 \pm 300 \mathrm{~K}$ (see Fig. 6 of Fontaine et al. 2013) and results in surface layers which are strongly coupled to the central thermal reservoir via an almost adiabatic convection zone. In such a scenario, Chen \& Hansen (2012) hypothesised that following convective mixing the surface layers should experience an increase in effective temperature of $\approx 500 \mathrm{~K}$. Employing a Monte Carlo approach they found that the non-DA gap arose as a natural consequence of convective mixing in white dwarfs with effective temperatures $\approx 6000 \mathrm{~K}$. Whilst this model has not been well-constrained by observations, it provides an explanation for the feature we observe at $6500 \mathrm{~K}$ (Fig 8). In the future larger volumelimited samples will be needed to ascertain the statistical significance of this bump.

\subsection{Magnetism}

Table 4 summarises all white dwarfs with a spectral type indicating magnetism. It does not include WD 2150+591, a DAH white dwarf at $8.34 \mathrm{pc}$ which does not have an astrometric solution in Gaia DR2 (see Table A3). The top panel of Fig. 9 shows the distribution of magnetic white dwarfs in the $T_{\text {eff }}$ versus $\log g$ diagram. It excludes the DQpecP J101141.58+284559.07 for which we have no reliable Gaia atmospheric parameters. The results suggest that magnetic white dwarfs are more massive than the average. We also observe that many magnetic white dwarfs follow the crystallisation sequence (see Section 5.5).

Our volume-limited sample allows to look at statistics of field strength versus cooling age as done by Landstreet \& Bagnulo (2019) for the $20 \mathrm{pc}$ sample. The results are shown in the bottom panel of Fig. 9 for the northern hemisphere 40 pc sample. This excludes DQP J123752.23+415624.69 for which the magnetic field strength is unclear. We observe no obvious correlation between field strength and temperature, as was found by Landstreet \& Bagnulo (2019).

For statistics as a function of mass and temperatures, we limit ourselves to DA white dwarfs with $T_{\text {eff }}>5000 \mathrm{~K}$ for which Zeeman splitting can be detected. This is because most helium-rich atmospheres are of DC spectral type, for which magnetic field detection is difficult. Even spectropolarimetric observations are orders of magnitude less sensitive to magnetic fields in DC white dwarfs compared to DA spectral type (Landstreet \& Bagnulo 2019). Fig. 10 shows magnetic fraction histograms with $T_{\text {eff }}$ and $\log g$ as independent variables for hydrogen-atmosphere white dwarfs. For both parameters, number statistics are relatively poor given that the curves are based on only 24 magnetic hydrogenatmosphere white dwarfs within $40 \mathrm{pc}$. It is clear that larger samples will be needed to confirm any correlation of magnetism with temperature and mass, in addition to potential biases against the detection of small magnetic fields. We note that since more massive white dwarfs are intrinsically fainter, the tentative increase in incidence as a function of $\log g$ observed in the bottom panel of Fig. 10 can not easily be explained by observational biases. Overall, the $40 \mathrm{pc}$ sample provides strong evidence that magnetic white dwarfs are more massive than the average, with $\bar{M}=0.75 \mathrm{M}_{\odot}$ for $24 \mathrm{DAH}, \mathrm{DAP}$ and DAZH versus $\bar{M}=0.66 \mathrm{M}_{\odot}$ for 278 non-magnetic DA and DAZ white dwarfs above $5000 \mathrm{~K}$. A Kolmogorov-Smirnov test gives a p-value of much less than one per cent, meaning we can reject this being a random fluctuation.

We have employed non-magnetic models to fit Gaia colours of magnetic white dwarfs. This could lead to a systematic effect on their atmospheric parameters. However, we note that they align with the same crystallisation sequence as non-magnetic white dwarfs. This is expected as magnetic fields of $B<1000 \mathrm{MG}$ have little influence on the cooling process (Tremblay et al. 2015), suggesting that Gaia derived atmospheric parameters of magnetic white dwarfs are accurate to at least a few percent. To obtain a better estimate of the accuracy of the atmospheric parameters for magnetic white dwarfs, Figs. 11-12 show GALEX-Gaia and GaiaWISE colour-colour diagrams, respectively. It demonstrates that for the vast majority of the magnetic white dwarfs in the sample, the shape of the energy distribution is empirically similar to non-magnetic white dwarfs. In other words, magnetic white dwarfs can be fitted with a single white dwarf model from the near-UV to the near-IR. Since this range covers most of the emergent flux, it suggests that Gaia $T_{\text {eff }}$ and mass values for magnetic white dwarfs are similarly accurate as those of non-magnetic white dwarfs. We conclude that the previously claimed higher-than-average mass values of magnetic white dwarfs (Ferrario et al. 2015; Kawka 2020) is a robust result. 
Table 4. Magnetic White Dwarfs in the Gaia DR2 Northern 40 pc Sample

\begin{tabular}{|c|c|c|c|}
\hline WDJ name & $\mathrm{SpT}$ & $\begin{array}{l}\langle B\rangle \\
{[\mathrm{MG}]}\end{array}$ & Ref \\
\hline $001214.75+502520.74$ & $\mathrm{DAH}$ & 0.25 & Landstreet \& Bagnulo (2019) \\
\hline $024208.44+111233.00$ & DAH & 0.7 & Ferrario et al. (2015) \\
\hline $025959.15+081156.43$ & DAH & 0.1 & Ferrario et al. (2015) \\
\hline $030350.56+060748.75$ & DXP & 500 & Landstreet \& Bagnulo (20 \\
\hline $033320.37+000720.65$ & $\mathrm{DAH}$ & 850 & Ferrario et al. (2015) \\
\hline $051553.54+283916.81$ & DAH & 1.1 & Limoges et al. (2015) \\
\hline $053714.90+675950.51$ & DAH & 0.7 & Tremblay et al. (2020) \\
\hline $063235.80+555903.12$ & DAH & 1 & Tremblay et al. (2020) \\
\hline $064400.61+092605.76$ & DAH & 3.2 & Tremblay et al. (2020) \\
\hline $064926.55+752124.97$ & DAH & 9 & Tremblay et al. (2020) \\
\hline $073330.88+640927.44$ & DAP & 0.1 & Ferrario et al. (2015) \\
\hline $075959.58+433521.10$ & DCP & 200 & Ferrario et al. (2015) \\
\hline $084516.87+611704.81$ & $\mathrm{DAH}$ & 0.8 & Tremblay et al. (2020) \\
\hline $085830.87+412635.75$ & $\mathrm{DAH}$ & 3.4 & Ferrario et al. (2015) \\
\hline $091556.08+532523.86$ & DCP & 100 & Ferrario et al. (2015) \\
\hline $094846.64+242125.88$ & DAP & 670 & Ferrario et al. (2015) \\
\hline $101141.58+284559.07$ & DQpecP & 100 & Ferrario et al. (2015) \\
\hline $102907.46+112719.28$ & DAH & 18 & Ferrario et al. (2015) \\
\hline $123752.23+415624.69$ & DQP & - & Ferrario et al. (2015) \\
\hline $130841.20+850228.16$ & DAP & 4.9 & Landstreet \& Bagnulo (2019) \\
\hline $133059.42+302953.65$ & DZH & 0.7 & Ferrario et al. (2015) \\
\hline $151534.80+823028.99$ & DZH & 3 & Tremblay et al. (2020) \\
\hline $153505.81+124745.20$ & DZH & 0.3 & Bagnulo \& Landstreet (2019) \\
\hline $164057.15+534109.32$ & DAH & 13 & Ferrario et al. (2015) \\
\hline $165445.69+382936.63$ & DAZH & 0.1 & Ferrario et al. (2015) \\
\hline $165948.42+440104.04$ & DAH & 2.3 & Ferrario et al. (2015) \\
\hline $171450.80+391837.43$ & DAH & 1.3 & Ferrario et al. (2015) \\
\hline $174807.99+705235.92$ & DXP & 100 & Ferrario et al. (2015) \\
\hline $181608.87+245442.85$ & DAP & 15 & Ferrario et al. (2015) \\
\hline $183020.27+544727.21$ & DXP & 170 & Ferrario et al. (2015) \\
\hline $190010.25+703951.42$ & DAP & 320 & Landstreet \& Bagnulo (2019) \\
\hline $201222.27+311348.88$ & DBP & 520 & Ferrario et al. (2015) \\
\hline $204906.70+372814.05$ & DAP & 0.06 & Landstreet \& Bagnulo (2019) \\
\hline $215140.11+591734.85$ & DAH & 0.8 & Landstreet \& Bagnulo (2019) \\
\hline $233203.52+265846.12$ & DAH & 2.3 & Ferrario et al. (2015) \\
\hline
\end{tabular}

\subsection{Crystallisation}

Table 5 provides statistics for white dwarfs roughly defined to be on the crystallisation sequence (Tremblay et al. 2019b) from

$$
\begin{aligned}
& \log g_{\text {Gaia }}> \frac{\left[\left(T_{\text {eff,Gaia }}-5000\right) / 1000\right]^{0.95}}{10}+8.00 \text { and } \\
& \log g_{\text {Gaia }}<\frac{\left[\left(T_{\text {eff,Gaia }}-5000\right) / 1000\right]^{0.95}}{4}+8.00 \text { and } \\
& 6000<T_{\text {eff,Gaia }}[\mathrm{K}]<12000 .
\end{aligned}
$$

This empirically selected region of over-density is shown in Fig. 13. We apply a lower temperature limit because there is no distinct crystallisation sequence below $6000 \mathrm{~K}$, as it merges with the peak in the $\log g$ distribution, e.g. the large majority of these cool white dwarfs could have started the crystallisation process. The upper temperature limit is applied because it is difficult to empirically isolate a crystallisation sequence above that temperature, i.e. only four hot and massive white dwarfs are potentially on the sequence. By applying the upper temperature cut, we also have average temperatures that are more similar for crystallising white dwarfs and those not on the sequence. We note that this
Table 5. Statistics of Crystallising and Non-Crystallising White Dwarfs $\left(6000 \mathrm{~K}<T_{\text {eff }}<12000 \mathrm{~K}\right)$

\begin{tabular}{lll}
\hline- & Crystallisation Seq. & Not on Seq. \\
$\overline{\overline{\log g}\left[\mathrm{~cm} / \mathrm{s}^{2}\right]}$ & 8.37 & 8.13 \\
$\overline{T_{\text {eff }}}[\mathrm{K}]$ & 7460 & 7800 \\
$\overline{v_{\perp}}[\mathrm{km} / \mathrm{s}]$ & 40.3 & 39.8 \\
$\mathrm{~N}(\mathrm{DQ}) / N_{\text {tot }}$ & $1.6 \% \pm 2 \%$ & $6.4 \% \pm 2 \%$ \\
$\mathrm{~N}(\mathrm{DA}) / N_{\text {tot }}$ & $79 \% \pm 15 \%$ & $70 \% \pm 7 \%$ \\
$\mathrm{~N}($ Magnetic $) / N_{\text {tot }}$ & $18 \% \pm 6 \%$ & $8.5 \% \pm 2 \%$ \\
\hline
\end{tabular}

experiment is different to the comparison of white dwarfs that have a liquid interior and those that have crystallised. Only a handful of massive white dwarfs have a temperature above $6000 \mathrm{~K}$ and are likely to be fully crystallised, but in the present analysis those are included in the bin of objects not currently crystallising.

Table 5 demonstrates that white dwarfs on the crystallisation sequence are in most measurable quantities very similar to white dwarfs that are not yet at that stage or have already solidified. In particular, white dwarfs on the crystallisation sequence are overwhelmingly of DA spectral type as outlined in Tremblay et al. (2019b). By construc- 


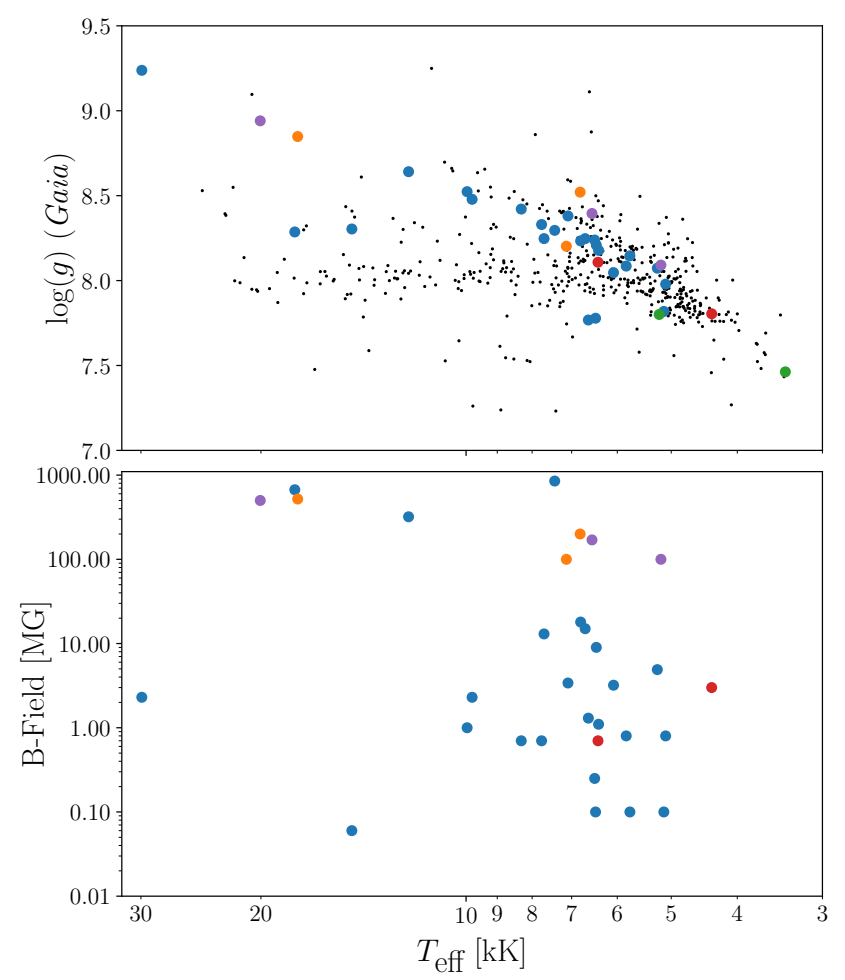

Figure 9. Top Panel: Atmospheric parameters of magnetic white dwarfs of different spectral types compared to the full 40 pc sample (black points). Bottom Panel: Magnetic field strength as a function of $T_{\text {eff }}$ for different spectral types. In both panels, $\mathrm{DAH} / \mathrm{DAP} / \mathrm{DAZH}$ are shown in blue, DCP/DBP in orange, DQP in green, DZH in red, and DX/DXP in purple.

tion white dwarfs with $T_{\text {eff }}>6000 \mathrm{~K}$ on the crystallisation are more massive than the average by about $0.12 \mathrm{M}_{\odot}$. Hence, any difference in their properties could be explained by their past evolution at these characteristic higher masses, and not necessarily by the crystallisation process itself. In particular, we detect a magnetic fraction that is marginally higher on the crystallisation sequence. Considering that the fraction of magnetic white dwarfs increases for the full sample as a function of mass (see Section 5.4), we speculate that there is no obvious causal link between magnetic field generation and crystallisation given the current sample size.

We find no significant difference between kinematic properties of crystallising white dwarfs and those that are not. This suggests that below $M \lesssim 1.0 \mathrm{M}_{\odot}$, there is no need to invoke a population of WD+WD mergers to explain the properties of crystallising white dwarfs (Cheng et al. 2019). This is a much different picture to the regime $M>1.08 \mathrm{M}_{\odot}$ studied in Cheng et al. (2019) and for which there is a significant difference between the kinematics of white dwarfs on the crystallisation sequence. In the northern $40 \mathrm{pc}$ sample, only 6 objects have $M>1.08 \mathrm{M}_{\odot}$ as a consequence of the steep initial mass function. Assuming that 20 per cent of those come from WD+WD mergers as suggested by Cheng et al. (2019), this would result, on average, in only a single example in our surveyed volume. Hence, such high-mass merger population would not produce any detectable signal in our $40 \mathrm{pc}$ sample. The fraction of white dwarfs that come
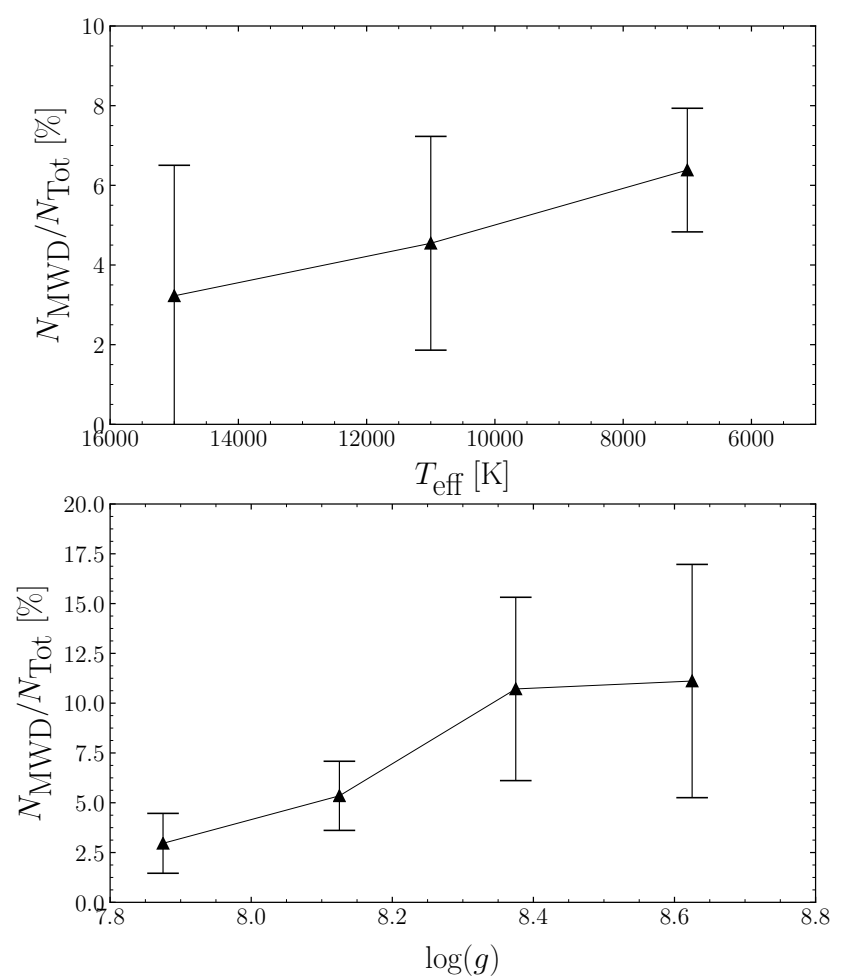

Figure 10. Top Panel: Magnetic incidence as a function of $\boldsymbol{T}_{\mathrm{eff}}$ for $\mathrm{H}$-atmosphere white dwarfs. Bottom Panel: Magnetic incidence as a function of $\log g$ for H-atmosphere white dwarfs.

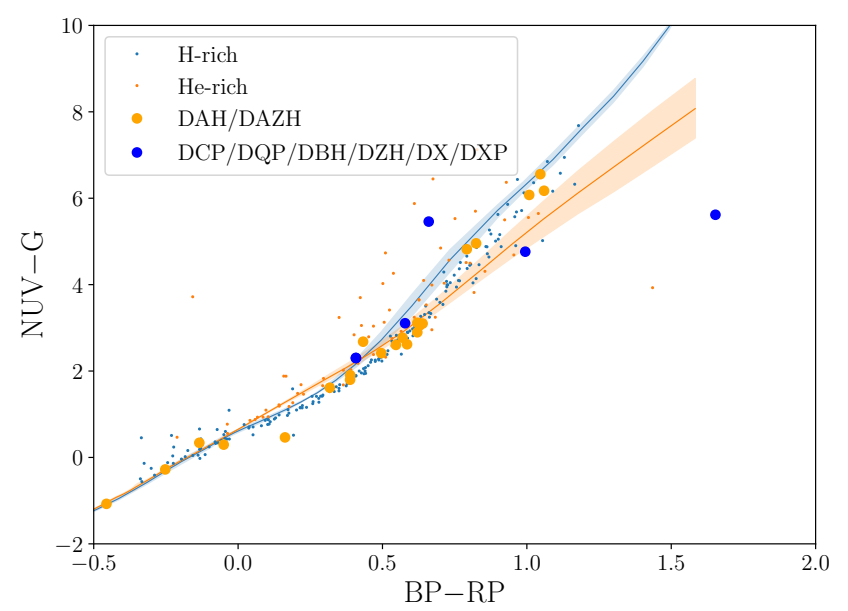

Figure 11. Colour-colour optical Gaia vs. GALEX NUV diagram highlighting magnetic white dwarfs with $\mathrm{H}$ - (blue) and He-rich (orange) composition. The shaded region indicates pure- $\mathrm{H}$ and pure-He model predictions for $7.5<\log g<8.5$ with $\log g=8.0$ shown with a darker line.

from mergers at lower masses is still an open issue which is difficult to quantify with our sample as these objects are likely to have measurable properties that are similar to white dwarfs that have evolved through single star evolution (Temmink et al. 2019). 


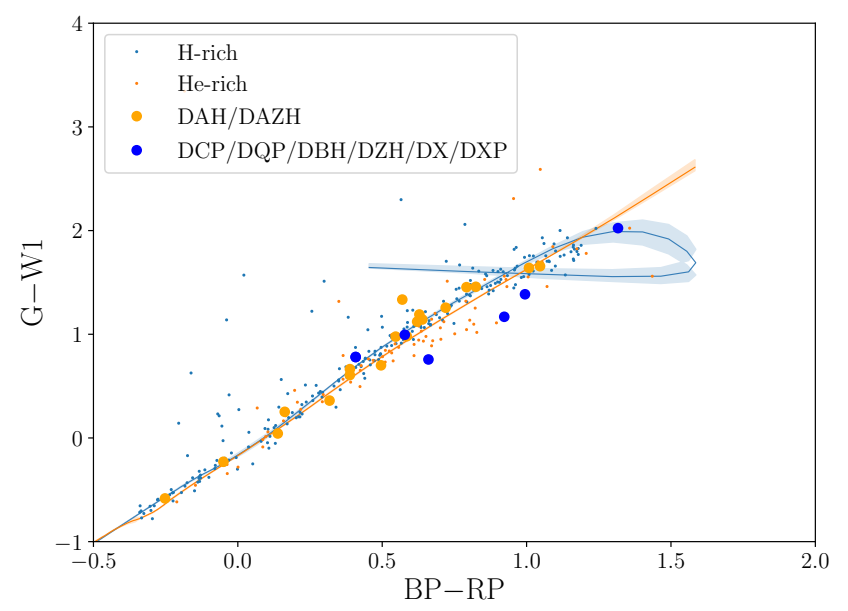

Figure 12. Colour-colour optical Gaia vs. WISE W1 near-IR diagram highlighting magnetic white dwarfs with $\mathrm{H}-$ (blue) and He-rich (orange) composition. The shaded region indicates pure$\mathrm{H}$ and pure-He model predictions for $7.5<\log g<8.5$ with $\log g=$ 8.0 shown with a darker line.

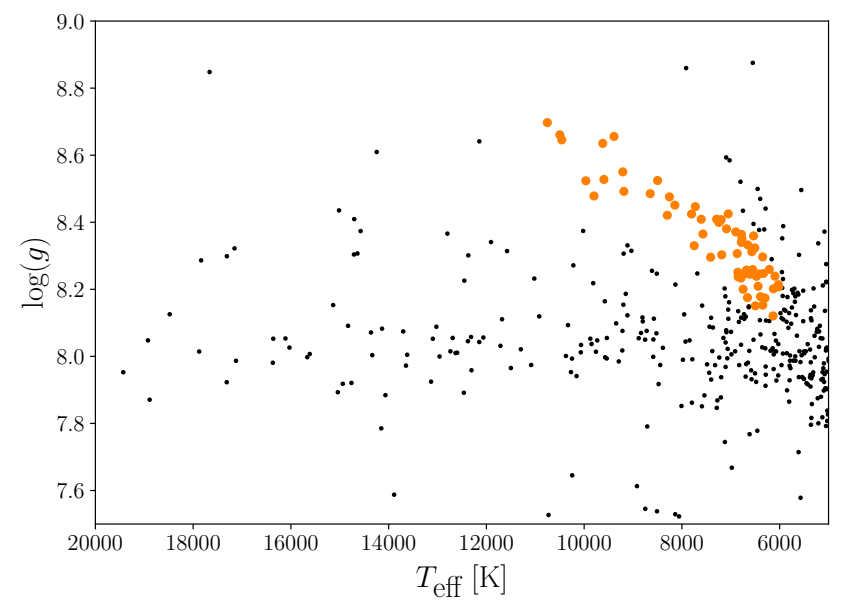

Figure 13. Empirically selected crystallisation sequence based on Eq. (2) and the Gaia $\log g$-temperature distribution.

\subsection{Ultra-cool white dwarfs}

Within our sample there are only four so-called ultra-cool white dwarfs $(\approx 1$ per cent), whose main feature is strongly non blackbody-like optical and near-IR colours due to CIA (Blouin et al. 2017). The location of these four ultra-cool white dwarfs in the Gaia HRD is shown in Fig. 14. These are among the faintest in the sample, lying below $G_{\text {abs }}=15.5$. However they do span a very wide range in Gaia colours, making it difficult to estimate the completeness of the current sample. The selection of Gentile Fusillo et al. (2019) managed to recover all four of the brightest previously known ultra-cool white dwarfs, suggesting that few additional objects are missing within 40 pc. Nevertheless, they lie in regions of the HRD where white dwarf identification is difficult due to a significant contamination from erroneous measurements. Two additional objects in the sample are possible

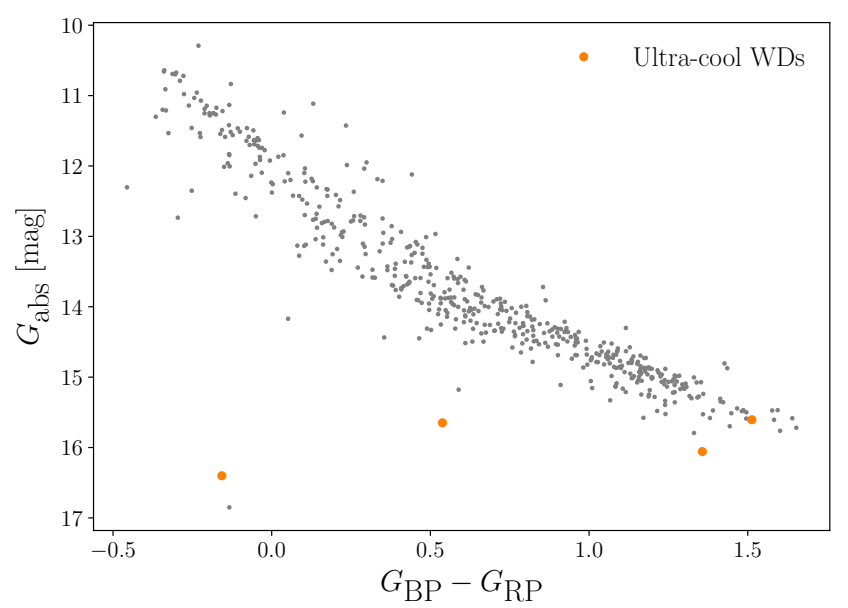

Figure 14. Position in the Gaia HRD of the four known ultracool white dwarfs with strong collision induced absorption and within the northern $40 \mathrm{pc}$ sample (orange points). The full northern $40 \mathrm{pc}$ white dwarf sample is shown in gray.

ultra-cool white dwarfs, the cool DZ J192206.20+023313.29 (see Paper I) and J050600.41+590326.89 for which spectroscopy is missing (see Section 2.2). Finally, we note that other objects in the sample show milder CIA in the near-IR. It is likely that this class of objects can not only be defined by surface temperature and chemical composition could also play a role (Kilic et al. 2020).

Table 6 shows the parameters of ultra-cool white dwarfs as found in the pre-Gaia literature, although caution should be used due to the uncertain nature of earlier CIA opacity calculations (see, e.g., Tremblay et al. 2014a). Curiously only two of the four objects have halo-like kinematics, while the other two are consistent with the Galactic disc (see Section 3). This could suggest that ultra-cool white dwarfs do not form a homogeneous Galactic population.

\section{CONCLUSIONS}

We have presented a review of the Gaia DR2 selected sample of white dwarfs within the $40 \mathrm{pc}$ northern hemisphere. This corresponds to the largest and most complete volume-sample of white dwarfs with low-resolution spectroscopic confirmation available so far, an increase of a factor of about four in number compared to the $20 \mathrm{pc}$ sample (Hollands et al. 2018 b). Our selection is based on the catalogue of Gentile Fusillo et al. (2019) while we have gathered spectral types from the literature and a companion paper (Tremblay et al. 2020). This results in a final sample of 524 white dwarfs, among which 521 have known spectral types. The existing observations are sufficient to determine the dominant atmospheric chemical constituent of individual white dwarfs (above $\approx 5000 \mathrm{~K}$ ), resulting in much better constraints on the atmospheric parameters compared to larger volume samples with low spectroscopic completeness. However, the varying quality of the spectroscopic signal-to-noise between observations prevents the derivation of robust absolute numbers of sub-types such as magnetic and metal-poluted white dwarfs. We note a systematic but moderate change in detection rates 
Table 6. Ultra-cool white dwarfs in the Gaia DR2 northern 40 pc sample. Error bars should be interpreted with caution because of systematic uncertainties in predicted CIA opacities.

\begin{tabular}{llllll}
\hline WDJ Name & SpT & $T_{\text {eff }}[\mathrm{K}]$ & $\log (\mathrm{g})$ & Mass $\left[\mathrm{M}_{\odot}\right]$ & Ref \\
\hline J034646.52+245602.67 & DC & $2970 \pm 40$ & $7.66 \pm 0.30$ & $0.39 \pm 0.10$ & Limoges et al. (2015) \\
J110217.52+411321.18 & DC & $3860 \pm 30$ & - & - & Limoges et al. (2015) \\
J165401.26+625354.91 & DC & $3080 \pm 100$ & $7.24 \pm 0.03$ & $0.22 \pm 0.01$ & Limoges et al. (2015) \\
J140324.75+453333.02 & DC & $2670 \pm 1500$ & - & - & Kilic et al. (2010) \\
\hline
\end{tabular}

of sub-types with distance, which can only be explained as an observational bias.

We find that most properties of the northern $40 \mathrm{pc}$ sample, such as the mean mass, kinematics, and fraction of wide and unresolved binaries are similar to those found for the four-time smaller $20 \mathrm{pc}$ sample, but some new trends appear in more rare subtypes of white dwarfs. We find a significantly lower mean mass for metal-rich DAZ white dwarfs compared to their parent DA population, which can not easily be explained as a simple statistical fluctuation. We suggest that it may inform about planet formation as a function of stellar mass (Veras et al. 2020). We find a significantly larger mean photometric mass for magnetic white dwarfs, which we determine is robust even though we rely on predicted magnitudes from non-magnetic models. Finally, the sample contains a notable sequence in $\log g-T_{\text {eff }}$ space of white dwarfs currently undergoing crystallisation (Tremblay et al. 2019b), which appear otherwise typical in all other measurable quantities.

The main advantage of the $40 \mathrm{pc}$ sample is that of better number statistics, possibly enabling the study of science questions that were not possible to answer with smaller samples. One example is the study of the local stellar formation history by relying on the reasonably well constrained white dwarf ages (Tremblay et al. 2014b; Fantin et al. 2019). The local white dwarf sample could also be combined with the full $40 \mathrm{pc}$ Gaia stellar sample to study overall stellar evolution, especially as new spectroscopic observations become available.

\section{ACKNOWLEDGEMENTS}

The research leading to these results has received funding from the European Research Council under the European Union's Horizon 2020 research and innovation programme n. 677706 (WD3D). This work presents results from the European Space Agency (ESA) space mission Gaia. Gaia data are being processed by the Gaia Data Processing and Analysis Consortium (DPAC). Funding for the DPAC is provided by national institutions, in particular the institutions participating in the Gaia MultiLateral Agreement (MLA). BTG has been supported by the STFC grant ST/T000406/1. ST acknowledge support from the Netherlands Research Council NWO (VENI 639.041.645 grants). ARM acknowledges support from the MINECO under the Ramón y Cajal programme (RYC-2016-20254), the AYA2017-86274-P grant and the AGAUR grant SGR-661/2017. PI acknowledges financial support from the Spanish Ministry of Economy and Competitiveness (MINECO) under the 2015 Severo Ochoa Programme MINECO SEV-2015-0548. The work presented made extensive use of TOPCAT (Taylor 2005).

\section{DATA AVAILABILITY STATEMENT}

The data underlying this article are available in the Gaia and Pan-STARRS public archives.

\section{REFERENCES}

Bagnulo S., Landstreet J. D., 2019, A\&A, 630, A65 Becklin E. E., Zuckerman B., 1988, Nature, 336, 656 Bédard A., Bergeron P., Fontaine G., 2017, ApJ, 848, 11 Bergeron P., Ruiz M. T., Leggett S. K., 1997, ApJS, 108, 339 Bergeron P., Leggett S. K., Ruiz M. T., 2001, ApJS, 133, 413

Bergeron P., et al., 2011, ApJ, 737, 28

Bergeron P., Dufour P., Fontaine G., Coutu S., Blouin S., GenestBeaulieu C., Bédard A., Rolland B., 2019, ApJ, 876, 67

Blouin S., Kowalski P. M., Dufour P., 2017, ApJ, 848, 36

Blouin S., Dufour P., Thibeault C., Allard N. F., 2019, ApJ, 878, 63

Brown J. M., Kilic M., Brown W. R., Kenyon S. J., 2011, ApJ, 730,67

Chambers K. C., et al., 2016, arXiv e-prints, p. arXiv:1612.05560

Chen E. Y., Hansen B. M. S., 2012, ApJ, 753, L16

Cheng S., Cummings J. D., Ménard B., 2019, ApJ, 886, 100

Coutu S., Dufour P., Bergeron P., Blouin S., Loranger E., Allard N. F., Dunlap B. H., 2019, ApJ, 885, 74

Crepp J. R., et al., 2018, ApJ, 864, 42

Cunningham T., Tremblay P.-E., Gentile Fusillo N. P., Hollands M., Cukanovaite E., 2020, MNRAS, 492, 3540

Dufour P., Blouin S., Coutu S., Fortin-Archambault M., Thibeault C., Bergeron P., Fontaine G., 2017, in Tremblay P. E., Gaensicke B., Marsh T., eds, Astronomical Society of the Pacific Conference Series Vol. 509, 20th European White Dwarf Workshop. p. 3

El-Badry K., Rix H.-W., 2018, MNRAS, 480, 4884

El-Badry K., Rix H.-W., Weisz D. R., 2018, ApJ, 860, L17

Fantin N. J., et al., 2019, ApJ, 887, 148

Ferrario L., de Martino D., Gänsicke B. T., 2015, Space Sci. Rev., 191,111

Fontaine G., Brassard P., Bergeron P., 2001, PASP, 113, 409

Fontaine G., Brassard P., Charpinet S., Rand all S. K., Van Grootel V., 2013, in European Physical Journal Web of Conferences. p. 05001, doi:10.1051/epjconf/20134305001

Fuchs B., Jahreiß H., 1998, A\&A, 329, 81

Gaia Collaboration et al., 2018a, A\&A, 616, A1

Gaia Collaboration et al., 2018b, A\&A, 616, A10

Genest-Beaulieu C., Bergeron P., 2019, ApJ, 871, 169

Gentile Fusillo N. P., Gänsicke B. T., Farihi J., Koester D., Schreiber M. R., Pala A. F., 2017, MNRAS, 468, 971

Gentile Fusillo N. P., Tremblay P. E., Jordan S., Gänsicke B. T., Kalirai J. S., Cummings J., 2018, MNRAS, 473, 3693

Gentile Fusillo N. P., et al., 2019, MNRAS, 482, 4570

Gentile Fusillo N. P., Tremblay P.-E., Bohlin R. C., Deustua S. E., Kalirai J. S., 2020, MNRAS, 491, 3613

Giammichele N., Bergeron P., Dufour P., 2012, ApJS, 199, 29

Gianninas A., Bergeron P., Ruiz M. T., 2011, ApJ, 743, 138 
Hall P. B., Kowalski P. M., Harris H. C., Awal A., Leggett S. K., Kilic M., Anderson S. F., Gates E., 2008, AJ, 136, 76

Hambly N. C., Smartt S. J., Hodgkin S. T., 1997, ApJ, 489, L157

Harris H. C., et al., 2013, ApJ, 779, 21

Haywood M., Di Matteo P., Lehnert M. D., Snaith O., Khoperskov S., Gómez A., 2018, ApJ, 863, 113

Helmi A., Babusiaux C., Koppelman H. H., Massari D., Veljanoski J., Brown A. G. A., 2018, Nature, 563, 85

Henry T. J., et al., 2018, AJ, 155, 265

Hirsch L. A., et al., 2019, ApJ, 878, 50

Holberg J. B., Oswalt T. D., Sion E. M., 2002, ApJ, 571, 512

Holberg J. B., Sion E. M., Oswalt T., McCook G. P., Foran S., Subasavage J. P., 2008, AJ, 135, 1225

Holberg J. B., Oswalt T. D., Sion E. M., Barstow M. A., Burleigh M. R., 2013, MNRAS, 435, 2077

Holberg J. B., Oswalt T. D., Sion E. M., McCook G. P., 2016, MNRAS, 462, 2295

Hollands M. A., Gänsicke B. T., Koester D., 2018a, MNRAS, 477, 93

Hollands M. A., Tremblay P. E., Gänsicke B. T., Gentile-Fusillo N. P., Toonen S., 2018b, MNRAS, 480, 3942

Isern J., 2019, ApJ, 878, L11

Jiménez-Esteban F. M., Torres S., Rebassa-Mansergas A., Skorobogatov G., Solano E., Cantero C., Rodrigo C., 2018, MNRAS, 480, 4505

Kawka A., 2020, arXiv e-prints, p. arXiv:2001.10672

Kawka A., Vennes S., 2006, ApJ, 643, 402

Kawka A., Vennes S., 2012, MNRAS, 425, 1394

Kawka A., Simpson J. D., Vennes S., Bessell M. S., Da Costa G. S., Marino A. F., Murphy S. J., 2020, arXiv e-prints, p. arXiv:2004.07556

Kilic M., et al., 2010, ApJS, 190, 77

Kilic M., Bergeron P., Dame K., Hambly N. C., Rowell N., Crawford C. L., 2019, MNRAS, 482, 965

Kilic M., Bergeron P., Kosakowski A., Brown W. R., Agueros M. A., Blouin S., 2020, arXiv e-prints, p. arXiv:2006.00323

Koester D., Weidemann V., Zeidler E. M., 1982, A\&A, 116, 147

Koester D., Voss B., Napiwotzki R., Christlieb N., Homeier D., Lisker T., Reimers D., Heber U., 2009, A\&A, 505, 441

Kowalski P. M., Saumon D., 2006, ApJ, 651, L137

Landstreet J. D., Bagnulo S., 2019, A\&A, 628, A1

Landstreet J. D., Bagnulo S., 2020, A\&A, 634, L10

Leggett S. K., Ruiz M. T., Bergeron P., 1998, ApJ, 497, 294

Leggett S. K., et al., 2018, ApJS, 239, 26

Liebert J., Dahn C. C., Monet D. G., 1988, ApJ, 332, 891

Liebert J., Bergeron P., Schmidt G. D., Saffer R. A., 1993, ApJ, 418,426

Limoges M. M., Bergeron P., Lépine S., 2015, ApJS, 219, 19

MacDonald J., Vennes S., 1991, ApJ, 371, 719

Maxted P. F. L., Marsh T. R., Moran C. K. J., Han Z., 2000a, MNRAS, 314, 334

Maxted P. F. L., Marsh T. R., Moran C. K. J., 2000b, MNRAS, 319,305

Minchev I., Chiappini C., Martig M., 2013, A\&A, 558, A9

Morrell S., Naylor T., 2019, MNRAS, 489, 2615

Munn J. A., et al., 2017, AJ, 153, 10

Ourique G., Romero A. D., Kepler S. O., Koester D., Amaral L. A., 2019, MNRAS, 482, 649

Parsons S. G., et al., 2010, MNRAS, 407, 2362

Parsons S. G., Rebassa-Mansergas A., Schreiber M. R., Gänsicke B. T., Zorotovic M., Ren J. J., 2016, MNRAS, 463, 2125

Parsons S. G., et al., 2018, MNRAS, 481, 1083

Putney A., 1997, ApJS, 112, 527

Rebassa-Mansergas A., Ren J. J., Parsons S. G., Gänsicke B. T., Schreiber M. R., García-Berro E., Liu X. W., Koester D., 2016, MNRAS, 458, 3808

Rebassa-Mansergas A., et al., 2017, MNRAS, 472, 4193

Rolland B., Bergeron P., Fontaine G., 2018, ApJ, 857, 56
Seabroke G. M., Gilmore G., 2007, MNRAS, 380, 1348

Sion E. M., Greenstein J. L., Landstreet J. D., Liebert J., Shipman H. L., Wegner G. A., 1983, ApJ, 269, 253

Sion E. M., Holberg J. B., Oswalt T. D., McCook G. P., Wasatonic R., 2009, AJ, 138, 1681

Subasavage J. P., et al., 2017, AJ, 154, 32

Taylor M. B., 2005, in Shopbell P., Britton M., Ebert R., eds, Astronomical Society of the Pacific Conference Series Vol. 347, Astronomical Data Analysis Software and Systems XIV. p. 29

Temmink K. D., Toonen S., Zapartas E., Justham S., Gänsicke B. T., 2019, arXiv e-prints, p. arXiv:1910.05335

Toonen S., Hollands M., Gänsicke B. T., Boekholt T., 2017, A\&A, 602, A16

Torres S., Cantero C., Rebassa-Mansergas A., Skorobogatov G., Jiménez-Esteban F. M., Solano E., 2019, MNRAS, 485, 5573

Tremblay P. E., Bergeron P., 2008, ApJ, 672, 1144

Tremblay P. E., Ludwig H. G., Steffen M., Bergeron P., Freytag B., 2011, A\&A, 531, L19

Tremblay P. E., Ludwig H. G., Steffen M., Freytag B., 2013, A\&A, 559, A104

Tremblay P. E., Leggett S. K., Lodieu N., Freytag B., Bergeron P., Kalirai J. S., Ludwig H. G., 2014a, ApJ, 788, 103

Tremblay P. E., Kalirai J. S., Soderblom D. R., Cignoni M., Cummings J., 2014b, ApJ, 791, 92

Tremblay P. E., Fontaine G., Freytag B., Steiner O., Ludwig H. G., Steffen M., Wedemeyer S., Brassard P., 2015, ApJ, 812,19

Tremblay P. E., Cummings J., Kalirai J. S., Gänsicke B. T., Gentile-Fusillo N., Raddi R., 2016, MNRAS, 461, 2100

Tremblay P. E., Cukanovaite E., Gentile Fusillo N. P., Cunningham T., Hollands M. A., 2019a, MNRAS, 482, 5222

Tremblay P.-E., et al., 2019b, Nature, 565, 202

Tremblay P. E., et al., 2020, arXiv e-prints, p. arXiv:2006.00965

Veras D., Tremblay P.-E., Hermes J. J., McDonald C. H., Kennedy G. M., Meru F., Gänsicke B. T., 2020, MNRAS, 493, 765

Winters J. G., et al., 2020, arXiv e-prints, p. arXiv:2004.11225

Zuckerman B., Becklin E. E., Macintosh B. A., Bida T., 1997, AJ, 113, 764

Zuckerman B., Koester D., Reid I. N., Hünsch M., 2003, ApJ, 596,477

Zuckerman B., Melis C., Klein B., Koester D., Jura M., 2010, ApJ, 722, 725

van Altena W. F., Lee J. T., Hoffleit E. D., 1995, The general catalogue of trigonometric [stellar] parallaxes. New Haven, CT: Yale University Observatory

van Leeuwen F., 2007, A\&A, 474, 653

\section{APPENDIX A: ONLINE TABLES}

This paper has been typeset from a $\mathrm{T}_{\mathrm{EX}} / \mathrm{LAT}_{\mathrm{EX}}$ file prepared by the author. 
Table A1. The catalogue of 524 Gaia white dwarfs in the northern hemisphere and within 40 pc can be accessed online at this link. See Table 1 for content description.

Table A2. List of 64 main-sequence star contaminants and spectroscopically unobserved low probability white dwarf candidates within $40 \mathrm{pc}$, in the northern hemisphere and drawn from the selection of Gentile Fusillo et al. (2019). The data can be accessed online at this link. See Table 1 for content description.

Table A3. White dwarfs in the northern hemisphere that are likely within $40 \mathrm{pc}$ and missing from Table A1 and the input sample of Gentile Fusillo et al. (2019), sorting by increasing distance.

\begin{tabular}{|c|c|c|c|c|c|c|}
\hline Gaia DR2 ID & Name & Parallax [mas] & Ref & $\mathrm{SpT}$ & Ref & Note \\
\hline \multicolumn{7}{|c|}{ Confirmed 40 pc Members } \\
\hline - & WD $0736+053$ & $284.56 \pm 1.26$ & $(1)$ & DQZ & Limoges et al. (2015) & (a) \\
\hline 3320184202856027776 & WD $0553+053$ & $125.0 \pm 3.6$ & $(2)$ & DAH & Limoges et al. (2015) & (b) \\
\hline- & WD $1334+039$ & $121.4 \pm 3.4$ & $(2)$ & DA & Limoges et al. (2015) & (c) \\
\hline 975968340912004352 & WD $0727+482 \mathrm{~A}$ & $88.543 \pm 0.066$ & (3) & DA & Limoges et al. (2015) & (d) \\
\hline 975968340910692736 & WD $0727+482 B$ & $88.543 \pm 0.066$ & (3) & DA & Limoges et al. (2015) & (d) \\
\hline 3978879594463069312 & WD $1121+216$ & $74.4 \pm 2.8$ & $(2)$ & DA & Limoges et al. (2015) & (b) \\
\hline 1362295082910131200 & HD 159062 & $46.123 \pm 0.024$ & (3) & G9V+WD & Hirsch et al. (2019) & (e) \\
\hline 2274076301516712704 & WD $2126+734 \mathrm{~B}$ & $45.15 \pm 0.21$ & $(4)$ & $\mathrm{DC}$ & Zuckerman et al. (1997) & (f) \\
\hline 3701290326205270528 & WD $1214+032$ & $42.784 \pm 0.063$ & (4) & DA & Limoges et al. (2015) & (f) \\
\hline 3817534337626005632 & WD $1120+073$ & $31.23 \pm 0.80$ & (3) & $\mathrm{DC}$ & Limoges et al. (2015) & (d) \\
\hline 3920187251456355072 & WD $1153+135$ & $28.29 \pm 0.66$ & $(5)$ & $\mathrm{DC}$ & Leggett et al. (2018) & (b) \\
\hline 1962707287281651712 & PM J22105+4532 & $27.759 \pm 0.088$ & (4) & $\mathrm{DC}$ & Limoges et al. (2015) & (f) \\
\hline 307323228064848512 & WD $0108+277$ & $26.35 \pm 0.11$ & $(4)$ & DAZ & Kawka \& Vennes (2006) & (f) \\
\hline \multicolumn{7}{|l|}{ Unresolved Binaries } \\
\hline 1005873614079882880 & LHS 1817 & $61.13 \pm 0.15$ & (4) & $\mathrm{M} 4.5 \mathrm{~V}+\mathrm{WD}$ & Winters et al. (2020) & $(\mathrm{g})$ \\
\hline 1548104507825815296 & WD $1213+528$ & $34.834 \pm 0.032$ & (4) & $\mathrm{DA}+\mathrm{dM}$ & Limoges et al. (2015) & (g) \\
\hline 1550299304833675392 & WD $1324+458$ & $32.734 \pm 0.030$ & (4) & $\mathrm{M} 3 \mathrm{~V}+\mathrm{DA}$ & Parsons et al. (2010) & (g) \\
\hline 3845263368043086080 & WD $0911+023$ & $28.40 \pm 0.37$ & (4) & $\mathrm{B} 9.5 \mathrm{~V}+\mathrm{WD}$ & Holberg et al. (2013) & (g) \\
\hline 4478524169500496000 & HD 169889 & $28.30 \pm 0.07$ & $(4)$ & G9V+WD & Crepp et al. (2018) & (g) \\
\hline 3831059120921201280 & WD $1026+002$ & $25.064 \pm 0.060$ & $(4)$ & $\mathrm{DA}+\mathrm{dM}$ & Koester et al. (2009) & (g) \\
\hline \multicolumn{7}{|c|}{ Possible 40 pc Members } \\
\hline 4018536882933053056 & WD $1132+275$ & - & - & $\mathrm{DC}$ & Limoges et al. (2015) & (b) \\
\hline 1267487150183614976 & WD $1143+256$ & - & - & DA & Limoges et al. (2015) & (h) \\
\hline 2701893698904233216 & WD $2140+078$ & - & - & DA & Limoges et al. (2015) & (b) \\
\hline
\end{tabular}

References: (1) van Leeuwen (2007), (2) van Altena et al. (1995), (3) Gaia DR2 (companion), (4) Gaia DR2 (white dwarf), (5) Leggett et al. (2018).

Notes: (a) No Gaia detection due to saturation of Procyon A. (b) White dwarf does not have DR2 five-parameter astrometry. (c) No Gaia detection, noting that the white dwarf has an $\approx 4^{\prime \prime} \mathrm{yr}^{-1}$ proper motion. (d) White dwarf does not have DR2 five-parameter astrometry, but known companion does. (e) White dwarf resolved at 2."7 according to Hirsch et al. (2019) but no Gaia DR2 source detected corresponding to the white dwarf. (f) DR2 five-parameter astrometry available but white dwarf absent from Gentile Fusillo et al. (2019) because of missing or incorrect colours (large BP/RP excess factor). (g) Missing from Gentile Fusillo et al. (2019) because of the important flux contribution from the non-degenerate companion in the optical. (h) Distance estimate of $<40 \mathrm{pc}$ in Limoges et al. (2015) based on atmospheric parameters. Detected with the established proper motion, but with a parallax of $1.44 \pm 0.55$ mas. 
Table A4. Gaia white dwarf candidates in the northern hemisphere that may lie within 40 pc based on Gaia parallax errors. The upper rows in each section are the objects within $1 \sigma$, and the lower those which lie within $2 \sigma$.

\begin{tabular}{|c|c|c|c|c|c|}
\hline WDJ Name & Gaia DR2 ID & WD Name & Parallax [mas] & $\mathrm{SpT}$ & Ref \\
\hline \multicolumn{6}{|l|}{ Confirmed White Dwarfs } \\
\hline WDJ001339.15+001924.58 & 2545505281002947200 & WD $0011+000$ & $24.96(0.06)$ & DA & Gianninas et al. (2011) \\
\hline WDJ055231.03+164250.27 & 3349849778193723008 & - & $24.97(0.06)$ & DBA & Tremblay et al. (2020) \\
\hline WDJ080247.02+564640.62 & 1081514379072280320 & - & $24.87(0.21)$ & $\mathrm{DC}$ & Tremblay et al. (2020) \\
\hline WDJ102203.66+824310.00 & 1146403741412820864 & - & $24.95(0.13)$ & DA & Tremblay et al. (2020) \\
\hline WDJ134118.69+022737.01 & 3713218786120541824 & WD $1338+027$ & $24.88(0.13)$ & DQ & Kilic et al. (2010) \\
\hline WDJ171430.49+212710.45 & 4567158653660872064 & WD $1712+215$ & $24.96(0.06)$ & DC & Putney (1997) \\
\hline WDJ180218.60+135405.46 & 4496751667093478016 & - & $24.97(0.07)$ & DAZ & Tremblay et al. (2020) \\
\hline WDJ184733.18+282057.54 & 4539227892919675648 & - & $24.87(0.16)$ & $\mathrm{DC}$ & Tremblay et al. (2020) \\
\hline WDJ192206.20+023313.29 & 4288942973032203904 & - & $24.95(0.32)$ & $\mathrm{DZ}$ & Tremblay et al. (2020) \\
\hline WDJ202956.18+391332.20 & 2064272612307218176 & WD $2028+390$ & $24.96(0.04)$ & DA & Gianninas et al. (2011) \\
\hline WDJ221321.31+034911.08 & 2707796667595813248 & NLTT 53229 & $24.68(0.33)$ & $\mathrm{DC}$ & Limoges et al. (2015) \\
\hline WDJ235750.73+194905.90 & 2822330113802737408 & - & $24.92(0.12)$ & $\mathrm{DZ}$ & Tremblay et al. (2020) \\
\hline WDJ070845.80+204451.70 & 3366672379112835328 & PM J07087+2044 & $24.80(0.10)$ & DA & Limoges et al. (2015) \\
\hline WDJ112105.81+375615.39 & 763981296484951936 & CBS 429 & $24.91(0.06)$ & DA & Limoges et al. (2015) \\
\hline WDJ133359.84+001655.03 & 3662951038644235776 & WD $1331+005$ & $24.45(0.35)$ & DQ & Ferrario et al. (2015) \\
\hline WDJ134043.36+020348.30 & 3663664003222454528 & WD $1338+023$ & $24.69(0.16)$ & $\mathrm{DC}$ & Leggett et al. (2018) \\
\hline WDJ231845.10+123602.77 & 2811321837744375936 & WD $2316+123$ & $24.87(0.08)$ & DAH & Limoges et al. (2015) \\
\hline \multicolumn{6}{|c|}{ Main Sequence Contaminants } \\
\hline WDJ005645.62+551556.10 & 423445945315773440 & - & $24.36(0.89)$ & Star & Tremblay et al. (2020) \\
\hline WDJ134252.41+003312.28 & 3663164069021692800 & - & $24.19(0.96)$ & Star & Tremblay et al. (2020) \\
\hline \multicolumn{6}{|l|}{ Unobserved Objects } \\
\hline WDJ015348.12+654946.61 & 518443341936881664 & - & $22.90(2.49)$ & - & Low $P_{\mathrm{WD}}$ \\
\hline WDJ171605.95+190544.28 & 4548611473051368576 & - & $24.43(0.90)$ & - & Low $P_{\mathrm{WD}}$ \\
\hline WDJ181131.97+132601.58 & 4497270567861853440 & - & $24.52(0.50)$ & - & Low $P_{\mathrm{WD}}$ \\
\hline WDJ194052.95+170459.04 & 1823962663799965440 & - & $24.82(0.97)$ & - & Low $P_{\mathrm{WD}}$ \\
\hline WDJ194943.60+152641.56 & 1819762318874306048 & - & $24.53(0.55)$ & - & Low $P_{\mathrm{WD}}$ \\
\hline WDJ002955.72+472645.48 & 389482855766584704 & - & $22.91(1.30)$ & - & - \\
\hline WDJ050647.89+203014.55 & 3408829849653432576 & LP 416-350 & $24.76(0.15)$ & - & - \\
\hline WDJ055326.34+062759.60 & 3322605013926459520 & - & $24.30(0.54)$ & - & - \\
\hline WDJ112542.42+041318.02 & 3812805230740561280 & - & $21.88(2.10)$ & - & Low $P_{\mathrm{WD}}$ \\
\hline WDJ115016.52+154700.09 & 3924562444445708288 & - & $21.81(2.44)$ & - & Low $P_{\mathrm{WD}}$ \\
\hline WDJ122048.91+482912.98 & 1545564017495017088 & - & $22.91(1.29)$ & - & Low $P_{\mathrm{WD}}$ \\
\hline WDJ123010.77+100537.85 & 3903961547910932992 & - & $22.00(2.59)$ & - & Low $P_{\mathrm{WD}}$ \\
\hline WDJ174129.76+015632.44 & 4375912002010905600 & - & $23.77(0.93)$ & - & Low $P_{\mathrm{WD}}$ \\
\hline WDJ194553.70+180829.53 & 1824144048862367104 & - & $23.26(1.05)$ & - & Low $P_{\mathrm{WD}}$ \\
\hline
\end{tabular}

Table A5. The catalogue of 56 wide binary systems including a white dwarf in the northern hemisphere and within 40 pc can be accessed online at this link.

Note: (1) Found in El-Badry \& Rix (2018), (2) Found in Hollands et al. (2018b). 